\title{
European Registry on Helicobacter pylori management (Hp-EuReg): patterns and trends in first- line empirical eradication prescription and outcomes of 5 years and 21533 patients
}

Olga P Nyssen, ${ }^{1}$ Dmitry Bordin (1) ${ }^{2,3}{ }^{\text {Bojan Tepes, }}{ }^{4}$ Ángeles Pérez-Aisa, ${ }^{5}$ Dino Vaira, ${ }^{6}$ Maria Caldas, ${ }^{1}$ Luis Bujanda, ${ }^{7}$ Manuel Castro-Fernandez, ${ }^{8}$ Frode Lerang, ${ }^{9}$ Marcis Leja, ${ }^{10}$ Luís Rodrigo, $^{11}$ Theodore Rokkas, ${ }^{12}$ Limas Kupcinskas, $^{13}$ Jorge Pérez-Lasala, ${ }^{14}$ Laimas Jonaitis, ${ }^{13}$ Oleg Shvets, ${ }^{15}$ Antonio Gasbarrini, ${ }^{16}$ Halis Simsek, ${ }^{17}$ Anthony T R Axon, ${ }^{18}$ György Buzás, ${ }_{1}^{19}$ Jose Carlos Machado (1) , 20 Yaron Niv, ${ }^{21}$ Lyudmila Boyanova, ${ }^{22}$ Adrian Goldis, ${ }^{23}$ Vincent Lamy, ${ }^{24}$ Ante Tonkic, ${ }^{25}$ Krzysztof Przytulski, ${ }^{26}$ Christoph Beglinger, ${ }^{27}$ Marino Venerito, ${ }^{28}$ Peter Bytzer, ${ }_{1}^{29}$ Lisette Capelle, ${ }^{30}$ Tomica Milosavljević, $^{31}$ Vladimir Milivojevic, ${ }^{31}$ Lea Veijola, ${ }^{32}$ Javier Molina-Infante, ${ }^{33}$ Liudmila Vologzhanina, ${ }^{34}$ Galina Fadeenko, ${ }^{35}$ Ines Ariño, ${ }^{36}$ Giulia Fiorini, ${ }^{6}$ Ana Garre, ${ }^{1}$ Jesús Garrido, ${ }^{37}$ Cristina F Pérez, ${ }^{38}$ Ignasi Puig, ${ }^{39}$ Frederic Heluwaert, ${ }^{40}$ Francis Megraud, ${ }^{41}$ Colm O'Morain, ${ }^{42}$ Javier P Gisbert (D) , ${ }^{1}$ On behalf of the Hp-EuReg Investigators

- Additional material is published online only. To view please visit the journal online (http://dx.doi.org/10.1136/ gutjnl-2020-321372).

For numbered affiliations see end of article.

Correspondence to Dr Javier P Gisbert,

Gastroenterology Department, Hospital Universitario de La Princesa, Madrid, Spain; javier.p.gisbert@gmail.com

Received 13 April 2020 Revised 8 June 2020 Accepted 26 June 2020 Published Online First 21 September 2020

\section{Linked}

- http://dx.doi.org/10.1136/ gutjnl-2020-322385

Check for updates

(C) Author(s) (or their employer(s)) 2021. No commercial re-use. See rights and permissions. Published by BMJ.

To cite: Nyssen OP, Bordin D, Tepes B, et al. Gut

$2021: 70: 40-54$

\section{ABSTRACT}

Objective The best approach for Helicobacter pylori management remains unclear. An audit process is essential to ensure clinical practice is aligned with best standards of care.

Design International multicentre prospective noninterventional registry starting in 2013 aimed to evaluate the decisions and outcomes in $\mathrm{H}$. pylori management by European gastroenterologists. Patients were registered in an e-CRF by AEG-REDCap. Variables included demographics, previous eradication attempts, prescribed treatment, adverse events and outcomes. Data monitoring was performed to ensure data quality. Timetrend and geographical analyses were performed.

Results 30394 patients from 27 European countries were evaluated and 21533 (78\%) firstline empirical $H$. pylori treatments were included for analysis. Pretreatment resistance rates were $23 \%$ to clarithromycin, $32 \%$ to metronidazole and $13 \%$ to both. Triple therapy with amoxicillin and clarithromycin was most commonly prescribed (39\%), achieving $81.5 \%$ modified intention-to-treat eradication rate. Over $90 \%$ eradication was obtained only with 10 -day bismuth quadruple or 14-day concomitant treatments. Longer treatment duration, higher acid inhibition and compliance were associated with higher eradication rates. Time-trend analysis showed a region-dependent shift in prescriptions including abandoning triple therapies, using higher acid-inhibition and longer treatments, which was associated with an overall effectiveness increase (84\%-90\%).

Conclusion Management of $H$. pylori infection by European gastroenterologists is heterogeneous,

\section{Significance of this study}

What is already known on this subject?

- Helicobacter pylori affects billions of people worldwide and is the main cause of chronic gastritis, peptic ulcer disease and gastric cancer.

- The ideal regimen to treat the infection remains unclear after more than 30 years of experience.

What are the new findings?

- Triple therapy prescriptions (reporting cure rates of approximately $80 \%$ ) have decreased, especially in those regions with highclarithromycin resistance.

- Over $90 \%$ eradication was only obtained with 10-day bismuth quadruple therapies or 14-day concomitant treatment.

- From 2013 to 2018, the observed shift to longer treatment duration, higher acid inhibition and compliance provided an increase in the effectiveness.

How might it impact on clinical practice in the foreseeable future?

- The results of this study indicate that the management of $H$. pylori infection by European gastroenterologists is heterogeneous, frequently suboptimal and discrepant with current recommendations. Consensus guideline improvements are being slowly incorporated into the daily clinical practice, which emphasises the importance of regular medical education and the need of surveillance. 
suboptimal and discrepant with current recommendations. Only quadruple therapies lasting at least 10 days are able to achieve over $90 \%$ eradication rates. European recommendations are being slowly and heterogeneously incorporated into routine clinical practice, which was associated with a corresponding increase in effectiveness.

\section{INTRODUCTION}

Helicobacter pylori ( $H$. pylori) is a bacterial pathogen with a $50 \%$ worldwide prevalence, being the main cause of chronic gastritis, peptic ulcer disease and gastric cancer. However, the ideal strategy to manage $H$. pylori infection remains unclear. The diagnostic method, the use of culture and antibiotic susceptibility testing, the treatment to prescribe and the test to confirm eradication are debatable, and recommendations have changed over time. ${ }^{1-5}$ Currently, most treatments are prescribed on an empiric basis, unaware of the bacterial antibiotic resistance profile. Noteworthy, recommendations have changed over time, with a shift from triple to quadruple therapies in the last consensus conferences. ${ }^{56}$ Therefore, a continuous evaluation of practice outcomes using the different management options is required in order to achieve high-quality 'evidence-based medicine'.

It is now accepted that chronic colonisation by $H$. pylori is an infectious disease and should be managed as such. ${ }^{7}$ For this reason, an optimal anti-H. pylori regimen is currently defined as one that reliably offers a cure rate of at least $90 \%$, accepted as an arbitrary threshold. ${ }^{8}{ }^{9}$ Triple therapies, using clarithromycin and amoxicillin, are still the most commonly used firstline therapies in spite of their failure in $\geq 20 \%-30 \%$ of patients. Resistance to clarithromycin has been identified as one of the major factors affecting $H$. pylori eradication success, and the rate of resistance to this antibiotic is steadily increasing in many geographical areas. ${ }^{10}$ For this reason, non-bismuth quadruple regimen, comprising a proton pump inhibitor (PPI), amoxicillin, clarithromycin and a nitroimidazole, has more recently been used as first-line treatment, ${ }^{5611} 12$ and has improved the efficacy of triple therapy, ${ }^{13}$ although its efficacy is impaired when dual metronidazole-clarithromycin resistance is present.

Bismuth has a strong bacteriostatic effect unaffected by resistance and displays a beneficial synergy when combined with several antibiotics, allowing to overcome bacterial resistance. ${ }^{14} 15$ Thus, combinations containing bismuth may be promising options in settings where there are high, unknown or increasing $H$. pylori antibiotic resistance rates. Traditionally, bismuth has been prescribed in a quadruple regimen containing a PPI with tetracycline and metronidazole. However, the treatment schemes are complex, and bismuth salts and tetracycline are not available in many parts of the world; therefore, these drawbacks have caused a tendency to restrict its use to patients with penicillin allergy, or those who require rescue treatments after failure of a clarithromycin-containing first-line treatment. ${ }^{16}{ }^{17}$ The latest approach to $\mathrm{H}$. pylori eradication has been the addition of bismuth to the standard triple therapy containing clarithromycin and amoxicillin, and this has also achieved encouraging results. ${ }^{18}$

Considering these treatment combinations, and all of the possible optimisations that can be added (length of treatment, ${ }^{19}$ dose of PPI, ${ }^{20}$ among others), it is hard to decide which treatment will provide good results ( $\geq 90 \%$ cure rates) aligned with current recommendations and standards. Evidence from clinical trials will always be equivocal because it is impossible to perform a single randomised trial to evaluate all existing treatments. Network meta-analyses, however, may provide an acceptable pooled approach enabling analysis of combinations of data from several treatment trials. However, evidence derived from clinical trials may not be extrapolated to clinical practice, in which there are no restrictive inclusion criteria, and where available caretime per patient and patient follow-up are more limited. ${ }^{21}$

Finally, there is a general delay from publication of recommendations to their implementation in routine clinical practice, ${ }^{22}{ }^{23}$ in which sometimes they reach full penetration after being outdated. ${ }^{24}$ Therefore, scientists recommend long-term studies evaluating practice and outcome trends, and tools able to provide real-time data from real practice (local, regional and global). ${ }^{25}$

The European Registry on Helicobacter pylori management (Hp-EuReg) brings together information on the real clinical practice of a majority of European countries, including thousands of patients with different bacterial resistance patterns and treatment accessibility. For these reasons, our aim was to establish a largescale long-term prospective clinical practice study providing an overview of the current situation regarding $H$. pylori management. The study would allow not only continuous assessment on the integration of clinical recommendations agreed on medical consensus but also monitoring of the temporal trends of management options and outcomes. These evaluations were aimed to decide on the best possible treatment strategies for improvement (globally and locally) ensuring that routine clinical practice is aligned with best standards of care.

\section{METHODS}

\section{European Registry on H. pylori management}

The 'European Registry on H. pylori Management' (Hp-EuReg) is an international multicentre prospective non-interventional registry recording information of $H$. pylori infection management since May 2013. Detailed information can be found in the published protocol, ${ }^{26}$ and is summarised in online supplementary file 2 .

\section{Statistical analyses}

Continuous variables are presented as mean and SD. Qualitative variables are presented as absolute and relative frequencies with percentages (\%). Graphical representations are used to show temporal trends in prescriptions. In the multivariate analysis, the effect was evaluated by calculating OR) and 95\% CI. Statistical significance was considered at $\mathrm{p}<0.05$.

\section{Effectiveness analysis}

The main outcome, which is treatment eradication rate, was studied in three sets of patients as follows: Intention-to-treat (ITT) analysis included all patients registered up to December 2017, to allow at least a 6-month follow-up, and lost to follow-up cases were considered treatment failures. Per-protocol (PP) analysis included all cases that finished follow-up and had taken at least $90 \%$ of the treatment drugs, as defined in the protocol. A modified ITT (mITT) was designed aiming to reach the closest result to those obtained in clinical practice. This mITT included for analyses all cases that had completed follow-up (ie, a confirmatory test-success or failure-was available after eradication treatment). Overall (ITT, mITT and PP) analyses were performed jointly for patients treated empirically. Additional PP effectiveness analyses were performed separately in those patients with a result of in vitro susceptibility testing.

All 27 countries were clustered in five main regions based both on their geographical situation and the 2017 gross domestic product per capita (online supplementary file 3 ). 
More than 100 different treatment schemes were used as first-line treatment. They were pooled in 13 categories (online supplementary file 4).

Similarly, PPI data were standardised using the PPI acid inhibition potency as defined by Kirchheiner ${ }^{27}$ and Graham, ${ }^{28} 29$ classified as low, standard and high dose PPI (online supplementary file 5).

The relation between eradication rate and age, gender, diagnosis, treatment length, PPI dose and compliance was studied in the mITT population considering six treatment categories (online supplementary file 6).

Mixed logistic regression models were used in a three-step strategy: the null model, the global mixed model with interaction between compliance and treatment and the mixed effects logistic regression for each treatment (online supplementary file 7).

\section{RESULTS}

From May 2013 to June 2018, 30394 cases were registered in the Hp-EuReg from 27 countries (distribution of patients per country is shown in online supplementary table 1). From those,
$21533(91 \%)$ were first-line therapies included in the current analysis (figure 1). Most of them were empirically treated; however, in $11 \%$ of the cases, bacterial antibiotic resistance data were available and were evaluated separately.

\section{Geographical analysis}

The 21533 naïve patients were distributed in the following five geographical regions: east (3679), south-east (4299), south-west (10118), centre (1985) and north (1452). The baseline characteristics are shown in table 1. A preliminary inspection showed high heterogeneity of practice and outcomes between European regions. For instance, 7 -day treatment prescription was marginal in south-western Europe (1.7\%), while it was mostly prescribed in south-eastern $(60.0 \%)$ and northern $(53.9 \%)$ regions. Most common treatments were also region specific: triple therapies were favoured in most of Europe $(82 \%-88 \%$ in south-eastern and northern Europe, $67 \%$ in the east and 34\% in south-west), whereas quadruple therapies were preferred in south-western and central Europe (63\%-82\%). Results of an additional cluster comparison performed between regions and the highest

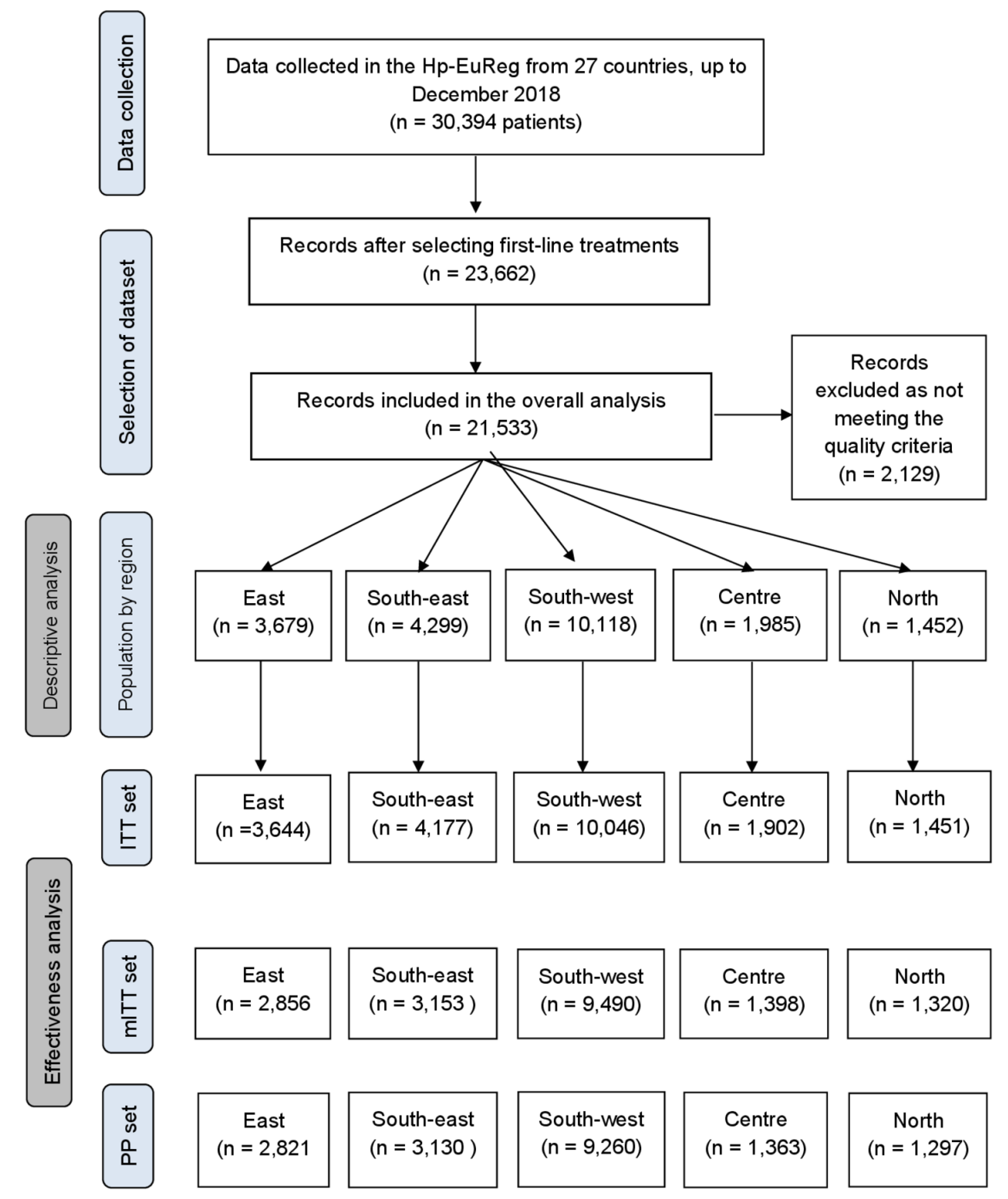

Figure 1 Study flow chart. ITT, intention-to-treat, mITT, modified ITT; PP, per-protocol. 
Table 1 Baseline characteristics of Helicobacter pylori first-line empirical treatments by region

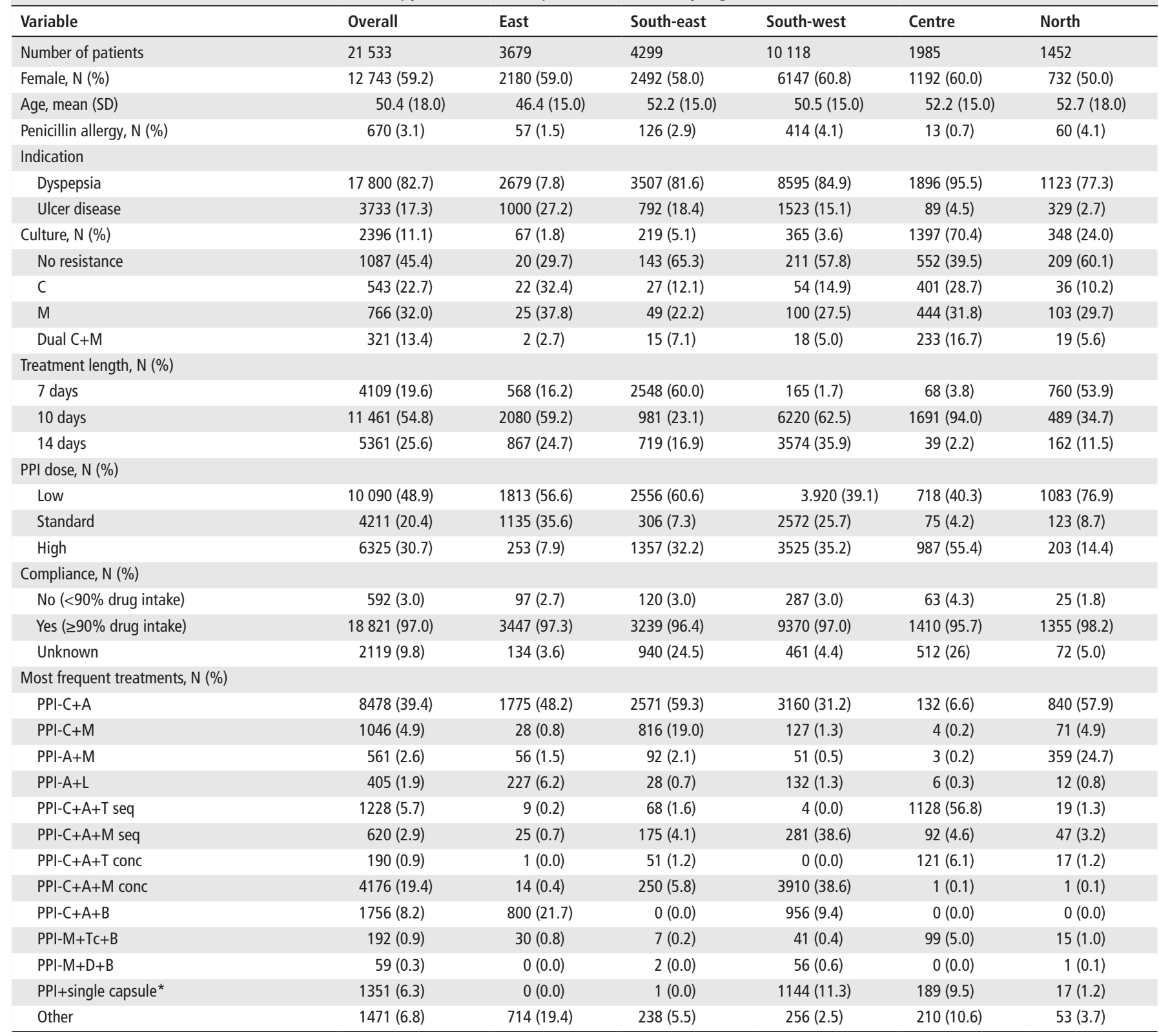

Low dose PPI: 4.5-27 mg omeprazole equivalents, two times per day (ie, $20 \mathrm{mg}$ omeprazole equivalents, two times per day), standard dose PPI: 32-40 mg omeprazole equivalents, two times per day (ie, 40 mg omeprazole equivalents, two times per day), high dose PPI: 54-128 mg omeprazole equivalents,two times per day (ie, $60 \mathrm{mg}$ omeprazole equivalents, two times per day).

* Three-in-one single capsule containing bismuth, tetracycline and metronidazole.

A, amoxicillin; B, bismuth salts; C, clarithromycin; Conc, concomitant; D, doxycycline; L, levofloxacin; M, metronidazole; PPI, proton pump inhibitor; Seq, sequential; T, tinidazole; Tc, tetracycline.

recruiting countries are presented in online supplementary table 2.

\section{Baseline characteristics}

Overall baseline characteristics, regional demographics and concomitant drug use are presented in table 1.

\section{Diagnosis}

Methods used for diagnosis of the infection and confirmation of eradication are detailed in online supplementary file 8 .

\section{Temporal trend analysis}

Figure 2A shows the prescription trends in Europe, where prescription shifts were region dependent: triple therapies did nearly disappear in south-western and central Europe, while they remained in the east, south-east and north. Triple therapies decreased from over $50 \%$ of prescription in 2013/2015 to less than $32 \%$ in $2017 / 2018$. Sequential therapies were prescribed in $8 \%$ in 2013 but yearly prescriptions were reduced up to $0.5 \%$ in 2018 , and concomitant therapy from $21 \%$ in $2013 / 2014$ to $11 \%$ in 2018. Use of bismuth quadruple therapies increased from $0 \%-2 \%$ in $2013 / 2014$ to $20 \%$ in 2018.

Figure $2 \mathrm{~B}$ depicts the trends on treatment duration, showing an increase in mean duration of treatments from 9.6 days in 2013, to 9.7 in 2014, 10.0 in 2015, 11.0 in 2016, 11.8 in 2017 and 11.8 days in 2018 , with regional differences. A major change that appeared to consistently occur throughout Europe was the discontinuation of 7-day therapies, especially in south-eastern 


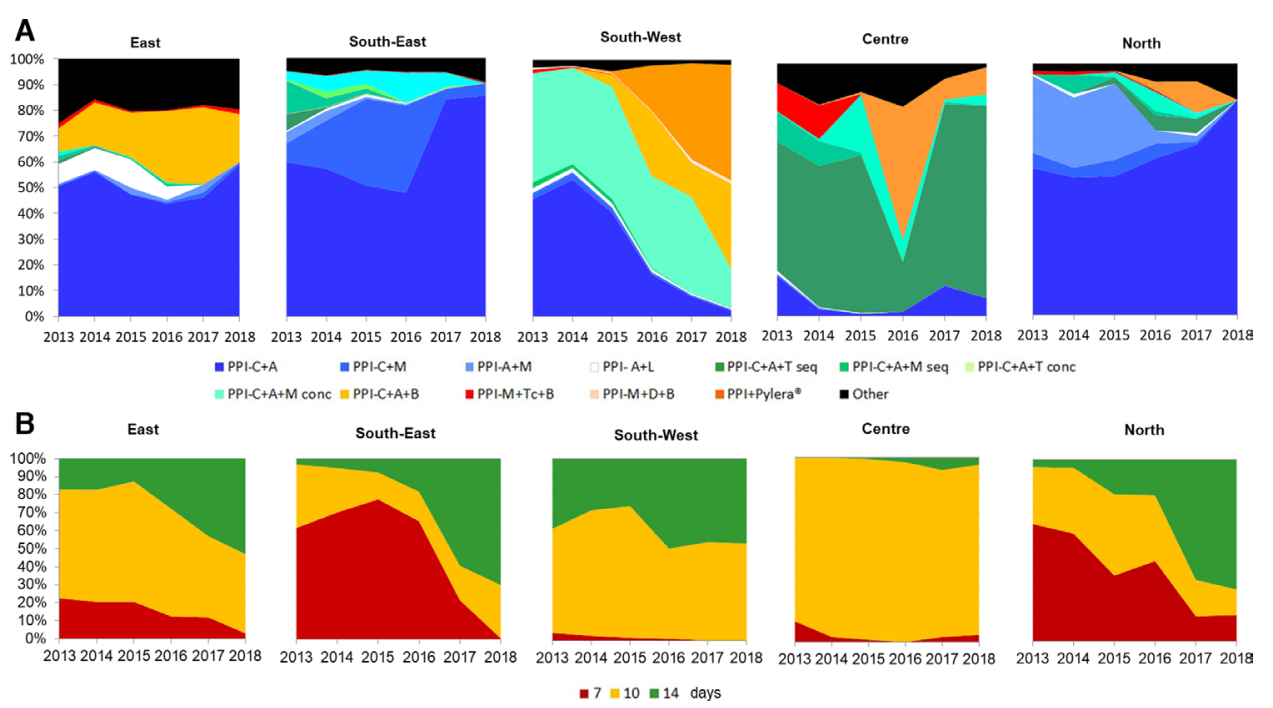

Figure 2 Treatment trends (2013-2018) in Europe per region. (A) Trends in the prescription of treatments. (B) Trends in the duration of treatments. A, amoxicillin; B, bismuth salts; C, clarithromycin; Conc, concomitant; D, doxycycline; L, levofloxacin; M, metronidazole; PPI, proton pump inhibitor; Seq, sequential; T, tinidazole; Tc, tetracycline.

and northern Europe, where it was still the most common therapy duration; however, 7-day therapies were scarcely used in other regions (online supplementary table 3 ).

Figure $3 \mathrm{~A}$ shows the trends in daily PPI dose (milligrams of omeprazole equivalent) by region and year, whereas figure $3 \mathrm{~B}$ shows the temporal trends in mean daily PPI dose. The potency of acid inhibition increased from a dose equivalent of $58 \mathrm{mg}$ of omeprazole in 2013 to $75 \mathrm{mg}$ in 2018, showing differences between regions. Mean daily dose of PPI increased in all regions except in central Europe where it decreased in 2017-2018. High doses of PPI were mainly used in south-eastern, south-western and northern Europe (online supplementary table 3).

\section{Treatment use and overall effectiveness}

Overall eradication rate increased from 2013 to 2018 independently of the population analysed:

- ITT: 70.1\% (2013), 72.6\% (2014), 74.5\% (2015), 76.7\% (2016), 75.2\% (2017), 77.3\% (2018).

- PP: 84.5\% (2013), 85.1\% (2014), 85.7\% (2015), 87.4\% (2016), 88.6\% (2017), 88.1\% (2018).

- mITT: 83.9\% (2013), 84.5\% (2014), 85.2\% (2015), 86.8\% (2016), 88.3\% (2017), 87.8\% (2018).

The effectiveness trends were region-specific (figure 4): Eastern Europe reported eradication rates lower than 70\% in 2013 and 2014, but achieved 80\% mITT in the following years. The remaining regions reported an overall treatment effectiveness higher than $80 \%$ in 2013. These rates increased in south-eastern and south-western countries, but remained constant in the centre and north.

The effectiveness trends also appeared to be treatment dependent in each region (table 2).

Triple therapy with clarithromycin and amoxicillin was the most frequent treatment in all regions but its eradication rate remained below $86.6 \%$ by mITT. None of the 12 treatments considered, except the concomitant therapy with clarithromycin, amoxicillin and tinidazole in the south-east, reached $90 \%$ effectiveness by mITT, whereas quadruple treatments achieved nearly 90\% eradication rate. In general, single capsule bismuth quadruple treatment was the most successful, achieving approximately $90 \%$ mITT eradication in those regions where it was prescribed.

The effectiveness was likewise modified depending on the duration of treatment. Table 3 shows the impact of treatment duration (7, 10 or 14 days). Overall, effectiveness increased with longer treatment duration; and this was mostly marked with specific treatments, such as the triple therapy with clarithromycin and amoxicillin or when bismuth was added to this triple regimen.

An additional univariate subanalysis was performed to evaluate the effect of standard (recommended) or high dose PPI in those 14-day treatments not reaching 90\% effectiveness (table 3).
A
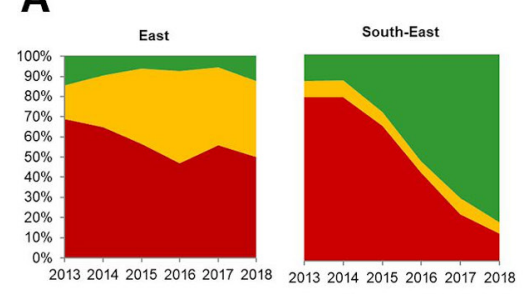

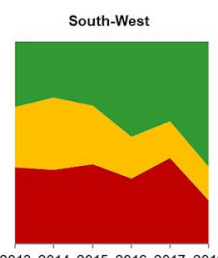

"low $\|$ standard $\|$ high PPI dose

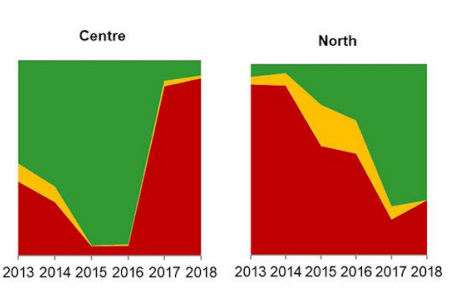

(1)
B

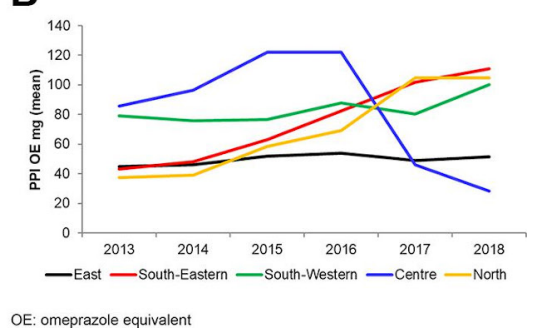

Figure 3 Trends (2013-2018) in the use of proton pump inhibitors (PPIs) in Europe per region. (A) Trends in the daily dose (low, standard and high) of PPI. (B) Trends in the mean daily dose of PPI. 


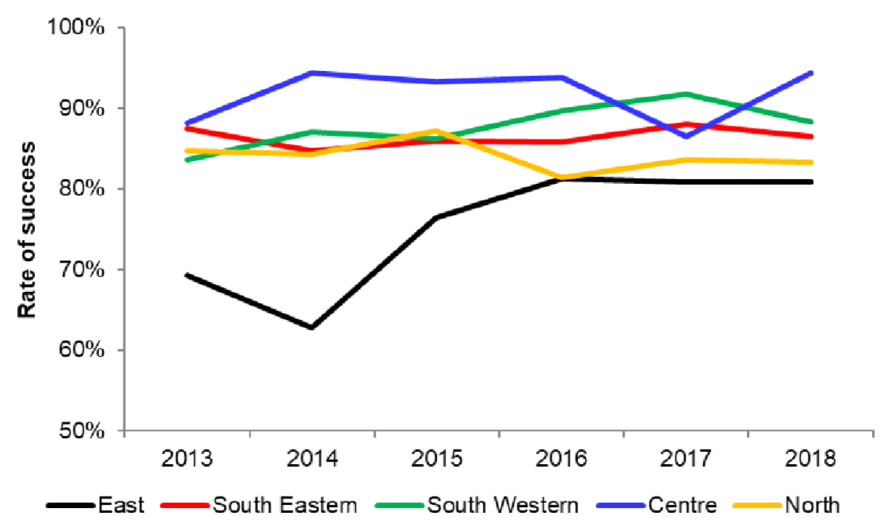

Figure 4 Trends in the eradication rate (modified intention-to-treat) by region.

\section{Resistance rates}

Data on susceptibility tailored prescription of antibiotics are presented in Table 1 and supplementary file 9.

The effect of resistance on eradication rate in the most frequent first-line treatments is reported in online supplementary table 4.

\section{Mixed effects logistic regression}

Null model

A null model without explanatory variables was developed to assess the proportion of variance of the outcome explained by grouping the cases in a second level 'centre'. There were 163 centres with an average of 89 cases per group. The variance of the intercept at centre level was 0.556 (SE 0.110) on the logit scale, and intraclass correlation coefficient (ICC) was 0.145 ; meaning the $14.5 \%$ of the variance of mITT effectiveness was explained by the differences between centres.

\section{Global mixed effects model with interaction between compliance and treatment}

The global mixed effects model showed a significant effect of compliance, with an OR of 6.8 (4.1 to 11.3), as well as an effect of treatment on mITT effectiveness. Using quadruple therapies with a PPI-clarithromycin-amoxicillin-bismuth as the reference category, ORs $(95 \% \mathrm{CI})$ were as follows: triple with clarithromycin-amoxicillin 0.494 (0.39 to 0.622$)$, triple with clarithromycin-metronidazole 0.220 (0.156 to 0.311$)$, sequential with clarithromycin-amoxicillin-metronidazole/tinidazole 0.452 (0.305 to 0.669), concomitant with clarithromycinamoxicillin-metronidazole/tinidazole 1.130 (0.879 to 1.453$)$, and the single capsule bismuth quadruple 1.766 (1.240 to $2.516)$, showing significantly higher mITT eradication rates in quadruple therapies compared with triple or sequential therapies. The interaction between compliance and treatment was significant $(p=0.02)$, showing that the difference in eradication rate between compliant and non-compliant patients changed from treatment to treatment. The interaction between compliance and treatments in terms of effectiveness is plotted in figure 5, which shows that independently of the treatment considered, in compliant patients, the rate of eradication (ranging between $80 \%$ and $95 \%$ ) was always higher compared with non-compliant patients. The effect of non-compliance on the mITT eradication rate was lower in concomitant therapy with clarithromycinamoxicillin-metronidazole/tinidazole and quadruple therapy with a PPI-clarithromycin-amoxicillin-bismuth than in the remaining treatments.

\section{Mixed effects logistic regression by treatment}

The final mixed effects logistic regression models were different for each treatment considered. To compare treatments easily, a tabular summary was built detailing the first level independent variables, the random variance component and ICCs for each model (table 4), whereas the final models are described in online supplementary file 10 .

\section{DISCUSSION}

In the present manuscript, we analysed the changes in $\mathrm{H}$. pylori treatment outcomes throughout a period of 5 years (2013-2018) across Europe. We found gastroenterologists' management of $H$. pylori in Europe is extremely heterogeneous (over 100 different first-line schemes), but a set of standard treatment schemes are most widely used: two-thirds as triple therapies and a quarter as quadruple therapies, and both generally prescribed as 10-day regimens. Moreover, our data show that there are strong regional differences in practice among European gastroenterologists. In this sense, our study shows that the generally unrecommended triple therapies have been abandoned in southern Europe, and are disappearing in eastern regions; however, their use is still widespread in central and northern Europe. This finding evidences an incomplete penetration or implementation of the last consensus conferences, which recommended switching from triple to quadruple therapies. ${ }^{4-6}$ This lack of update in clinical practice causes a high rate of failures ( $>20 \%$ in those patients), far from the current arbitrary threshold for acceptance of a proposed treatment ( $\geq 90 \%$ eradication rate). ${ }^{6} 89$

However, our trend analyses of first-line prescriptions showed that European gastroenterologists are at last adapting their practice to recommendations: some regions have dropped or are abandoning triple therapies, causing an overall drop of prescriptions from $50 \%$ to less than 20\%. Furthermore, central, northern and south-western Europe are increasing PPI dose and lengthening treatment duration (7-day therapies have decreased from one-third of prescriptions to less than $1 \%$, being currently marginal in all regions). As expected, this improvement in adherence to guidelines and recommendations has correlated with an improvement in efficacy rates, reaching in 2018 almost the proposed minimum $90 \%$ cure rate by ITT.

This manuscript focused on the most relevant first-line treatments used according to either their popularity or their success rate. The most commonly prescribed treatment was standard triple therapy with a PPI, clarithromycin and amoxicillin, achieving lower than $90 \%$ eradication rate even when given for 14 days, as previously described in the literature. ${ }^{3031}$ Moreover, in our study this treatment was greatly affected by clarithromycin resistance, reducing the effectiveness to below $50 \%$ in patients harbouring resistant strains. In this respect, pretreatment clarithromycin resistance in our study was $23 \%$, quite higher than the $15 \%$ resistance threshold generally considered (although the number of patients with susceptibility testing in the Hp-EuReg was very limited). However, triple therapy could still be used in those areas with low clarithromycin resistance and proven high effectiveness.

One of the proposed options chosen to improve triple therapy has been to combine PPI, amoxicillin, clarithromycin and metronidazole in one single scheme, the so-called 'non-bismuth quadruple treatment', given as either a sequential or concomitant regimen. Non-bismuth quadruple sequential treatment comprises PPI with amoxicillin combined during a first phase, and a second phase with a PPI, clarithromycin and metronidazole, respectively, each phase lasting for at least 5 days. Although 
Table 2 Effectiveness of most common first-line empirical treatments per region

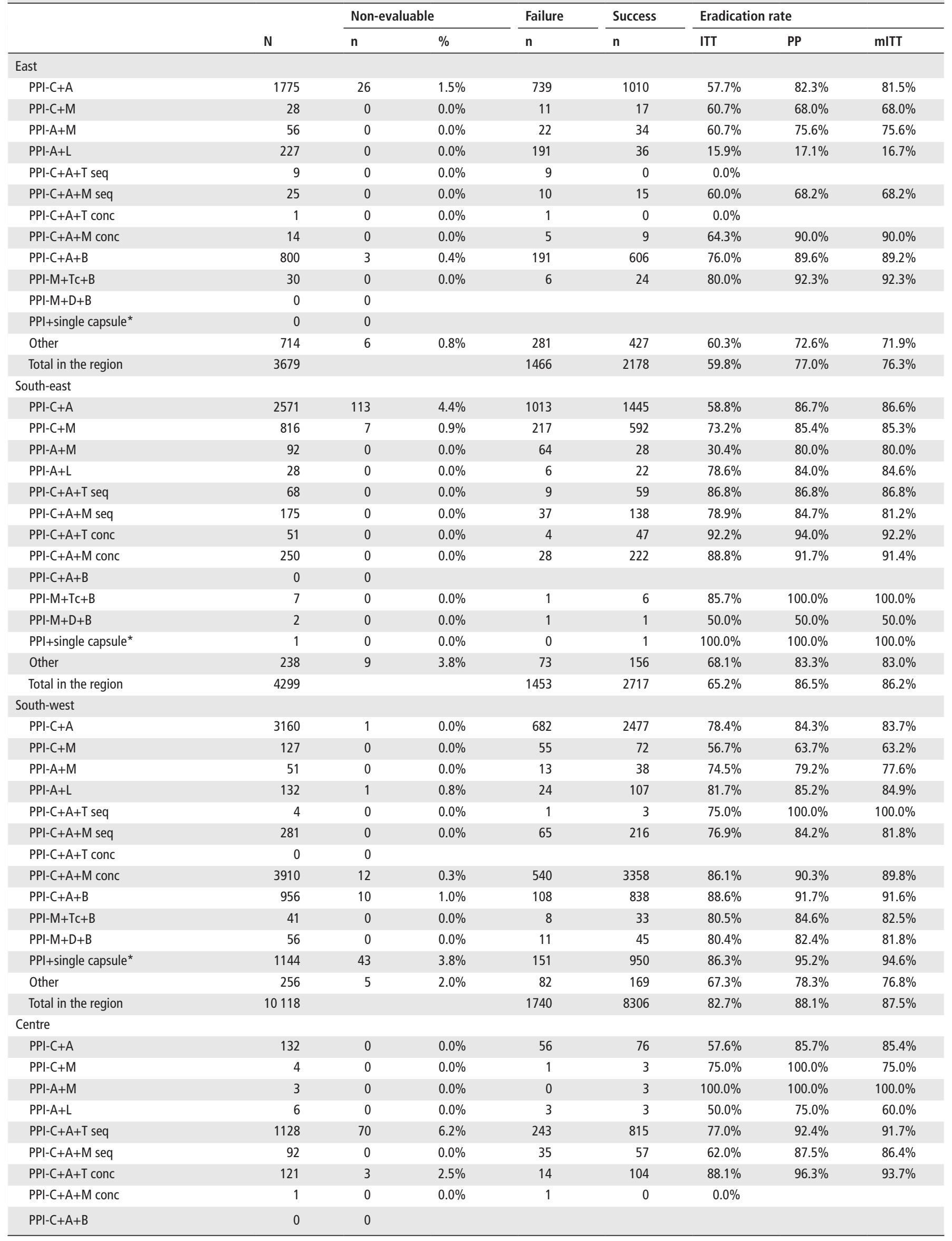


Table 2 Continued

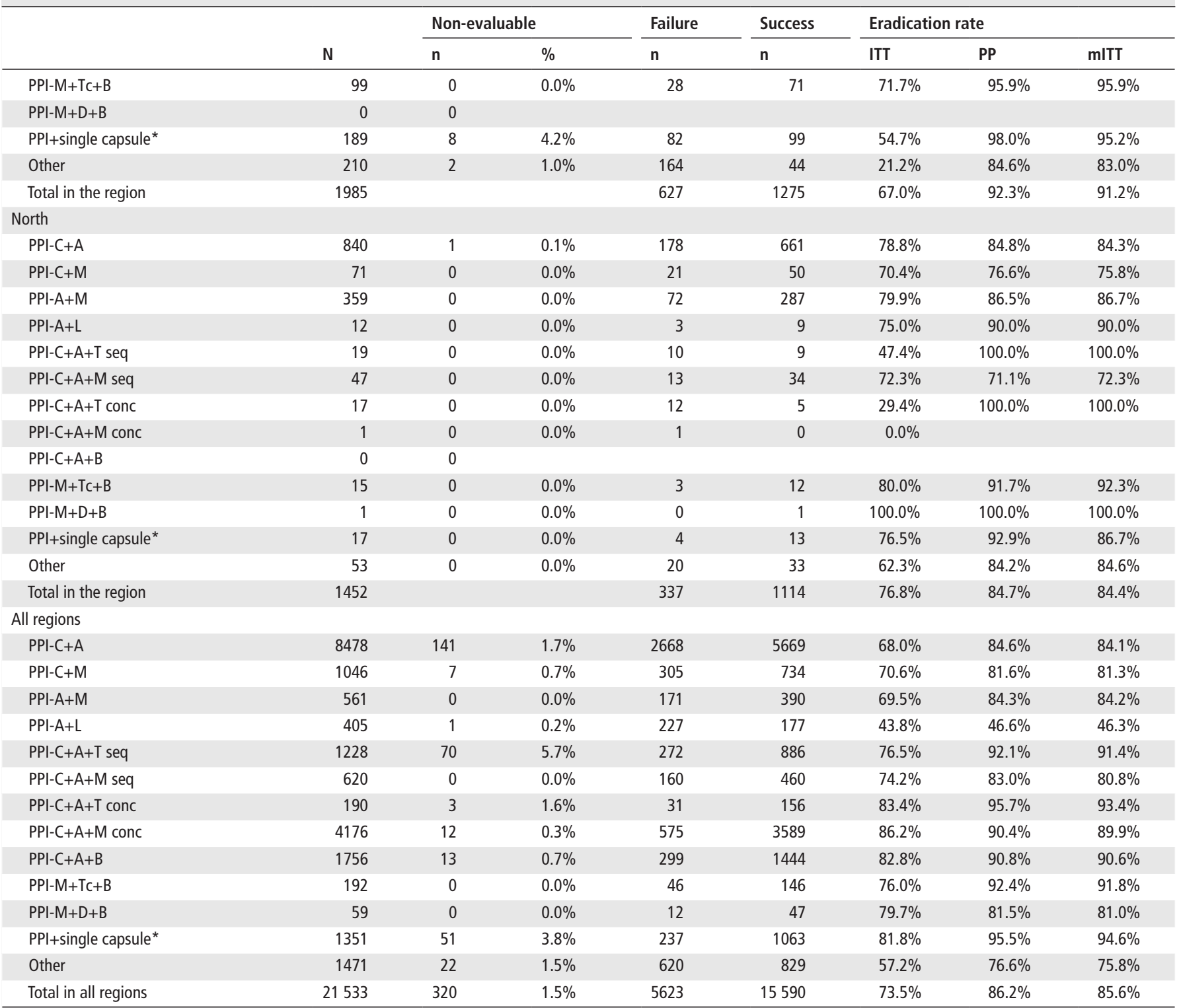

${ }^{*}$ Three-in-one single capsule containing bismuth, tetracycline and metronidazole.

A, amoxicillin; B, bismuth salts; C, clarithromycin; Conc, concomitant; D, doxycycline; L, levofloxacin; M, metronidazole; mlTT, modified intention-to-treat; PP, per protocol; PPI, proton pump inhibitor; Seq, sequential; Tc, tetracycline.

it was successfully proposed and implemented in the early $2000 \mathrm{~s},{ }^{32}$ it has been falling into disuse and it accounted for less than $10 \%$ of first-line treatments in our study. The eradication rate of the sequential therapy in our study $(86 \%)$ was superior to that of triple therapies but still below $90 \%$. Furthermore, sequential therapy is affected by single and, especially, by dual resistance to clarithromycin and metronidazole,${ }^{13}$ scoring below $80 \%$ and $75 \%$ in single and dual resistances, respectively.

Non-bismuth quadruple concomitant therapy includes the same drugs as sequential therapy but they are all taken together, with benefits in terms of simplicity, for both patients and physicians. The literature is clear regarding its superiority to triple therapies and, although more debatable, it is better than the quadruple sequential therapy as well. ${ }^{12} 33-35$ Our study showed that concomitant regimen during 10 days with standard acid inhibition was still unable to reach the $90 \%$ cure rate arbitrary threshold, but optimised regimens lasting 14 days and/or using high dose PPIs did achieve over 90\% eradication even in clarithromycin-resistant strains.

Another treatment that has resurfaced recently in light of increased resistance rates is bismuth quadruple therapy, which contains a PPI, bismuth salts, tetracycline and metronidazole. ${ }^{34} 36$ This combination has been available as a rather complicated multiprescription regimen scheme for many years, but recently, a three-in-one single, combination treatment has emerged. ${ }^{37}$ In the literature, both the traditional and the single capsule bismuth quadruple regimens achieve eradication rates near or superior to $90 \%$ irrespective of clarithromycin resistance, and even overcoming metronidazole resistance. ${ }^{37-39}$ In our study, they both achieved this threshold in 10-day treatments, even though the treated population was biased towards a higher prevalence of penicillin allergy, which has been proposed to be a risk factor for treatment failure. ${ }^{40}$ As in previous literature, ${ }^{37}$ metronidazole resistance did not significantly affect these bismuth quadruple regimens in our study. 
Table 3 Effectiveness of first-line empirical treatments according to duration

\begin{tabular}{|c|c|c|c|c|c|c|c|c|c|c|c|c|}
\hline & \multicolumn{4}{|c|}{ ITT eradication rate } & \multicolumn{4}{|c|}{ PP eradication rate } & \multicolumn{4}{|c|}{ mITT eradication rate } \\
\hline & \multirow[b]{2}{*}{$\mathrm{N}$} & \multicolumn{3}{|c|}{ Treatment duration } & \multirow[b]{2}{*}{ N } & \multicolumn{3}{|c|}{ Treatment duration } & \multirow[b]{2}{*}{ N } & \multicolumn{3}{|c|}{ Treatment duration } \\
\hline & & 7 days & 10 days & 14 days & & 7 days & 10 days & 14 days & & 7 days & 10 days & 14 days \\
\hline $\mathrm{PPI}-\mathrm{C}+\mathrm{A}^{*}$ & 8337 & $60.4 \%$ & $71.5 \%$ & $73.2 \%$ & 6647 & $83.0 \%$ & $84.8 \%$ & $86.7 \%$ & 6743 & $82.7 \%$ & $84.2 \%$ & $86.2 \%$ \\
\hline $\mathrm{PPI}-\mathrm{C}+\mathrm{M}^{*}$ & 1039 & $74.0 \%$ & $57.4 \%$ & $54.3 \%$ & 898 & $84.8 \%$ & $67.3 \%$ & $67.9 \%$ & 903 & $84.4 \%$ & $66.7 \%$ & $67.9 \%$ \\
\hline $\mathrm{PPI}-\mathrm{A}+\mathrm{M}$ & 561 & $69.3 \%$ & $77.4 \%$ & $25.0 \%$ & 458 & $80.7 \%$ & $85.9 \%$ & $80.0 \%$ & 463 & $80.8 \%$ & $85.7 \%$ & $80.0 \%$ \\
\hline PPI-A+L & 404 & $8.6 \%$ & $78.0 \%$ & $74.2 \%$ & 371 & $8.9 \%$ & $86.8 \%$ & $85.2 \%$ & 382 & $8.8 \%$ & $85.4 \%$ & $85.2 \%$ \\
\hline $\mathrm{PPI}-\mathrm{C}+\mathrm{A}+\mathrm{T}$ seq & 1158 & NA & $77.5 \%$ & NA & 957 & NA & $92.1 \%$ & NA & 969 & NA & $91.5 \%$ & NA \\
\hline $\mathrm{PPI}-\mathrm{C}+\mathrm{A}+\mathrm{M}$ seq ${ }^{*}$ & 620 & NA & $74.4 \%$ & NA & 528 & NA & $82.9 \%$ & NA & 569 & NA & $80.8 \%$ & NA \\
\hline $\mathrm{PPI}-\mathrm{C}+\mathrm{A}+\mathrm{T}$ conc & 187 & NA & $85.4 \%$ & $89.5 \%$ & 162 & NA & $95.5 \%$ & $100.0 \%$ & 167 & NA & $92.8 \%$ & $100.0 \%$ \\
\hline $\mathrm{PPI}-\mathrm{C}+\mathrm{A}+\mathrm{M}$ conc & 4164 & NA & $84.8 \%$ & $88.2 \%$ & 3891 & $95.0 \%$ & $88.9 \%$ & $92.2 \%$ & 3992 & $90.9 \%$ & $88.3 \%$ & $92.1 \%$ \\
\hline $\mathrm{PPI}-\mathrm{C}+\mathrm{A}+\mathrm{B}$ & 1743 & $50.0 \%$ & $74.6 \%$ & $86.6 \%$ & 1577 & $76.9 \%$ & $86.6 \%$ & $92.6 \%$ & 1594 & $76.9 \%$ & $86.2 \%$ & $92.4 \%$ \\
\hline $\mathrm{PPI}-\mathrm{M}+\mathrm{Tc}+\mathrm{B}$ & 192 & NA & $75.3 \%$ & $83.3 \%$ & 157 & NA & $93.8 \%$ & $88.2 \%$ & 159 & NA & $93.1 \%$ & $88.2 \%$ \\
\hline $\mathrm{PPI}-\mathrm{M}+\mathrm{D}+\mathrm{B}$ & 59 & NA & $93.8 \%$ & $78.0 \%$ & 54 & NA & $93.3 \%$ & $81.1 \%$ & 58 & NA & $93.8 \%$ & $80.0 \%$ \\
\hline PPI+single capsulet & 1300 & NA & $82.1 \%$ & NA & 1102 & NA & $95.4 \%$ & NA & 1124 & NA & $94.5 \%$ & NA \\
\hline Other & 1449 & $62.1 \%$ & $73.4 \%$ & $54.5 \%$ & 1073 & $73.7 \%$ & $85.0 \%$ & $60.4 \%$ & 1094 & $73.7 \%$ & $84.4 \%$ & $59.7 \%$ \\
\hline Total & 21213 & $61.1 \%$ & $76.9 \%$ & $79.6 \%$ & 17875 & $78.5 \%$ & $87.8 \%$ & $88.3 \%$ & 18217 & $78.2 \%$ & $87.0 \%$ & $88.0 \%$ \\
\hline
\end{tabular}

*An effectiveness univariate analysis was performed accounting 10-day or 14-day treatments prescribed together with high doses PPI only, and following therapies reached over 90\% mITT eradication rate: 14-day PPI-C+A (89.6\%), 10-day PPI-C+A+M seq (91.6\%), 10-day or14-day PPI-C+A+M conc (both 92.7\% and 92.8\%), 10-day PPI-C+A+B (95.5\%), 10-day PPI-M+Tc+B (95.2\%). A $\chi^{2}$ test was also performed and significant comparisons (10 days vs 14 days with high-dose PPIs) were reported in the table ( ${ }^{*}$ ). Additional pair-wise comparison (by means of $\mathrm{Chi}^{2}$ test and Fisher exact test) were performed between following treatments: 10-day sequential, 14-day concomitant and 10-day bismuth quadruple: statistically significant differences $(p<0.001)$ were found in all comparisons in favour of the 14-day concomitant and 10-day bismuth quadruple therapies. tThree-in-one single capsule containing bismuth, tetracycline and metronidazole.

A, amoxicillin; B, bismuth salts; C, clarithromycin; Conc, concomitant; ITT, intention-to-treat; L, levofloxacin; M, metronidazole; mITT, modified-intention-to treat; NA, Not applicable; PP, per protocol; PPI, proton pump inhibitor; Seq, sequential; Tc, tetracycline.

Finally, bismuth may also be combined with clarithromycin and amoxicillin to improve the efficacy of standard triple therapy. This approach has not been widely used in the literature, but a few studies have been published recently with encouraging results and have promoted a change in practice. ${ }^{4142}$ Our results with this treatment were also promising when prescribed for 14 days, scoring over $90 \%$ eradication by mITT.

In our mixed multilevel analysis, several factors were found to be independently associated with treatment effectiveness, especially adherence to treatment, with a global OR of approximately 7. Remarkably, compliance was excellent (97\%) and was indeed the factor which was mostly associated with higher eradication rate in all treatment categories evaluated, with an OR ranging from 4 (concomitant treatment) to 50 (quadruple therapy with

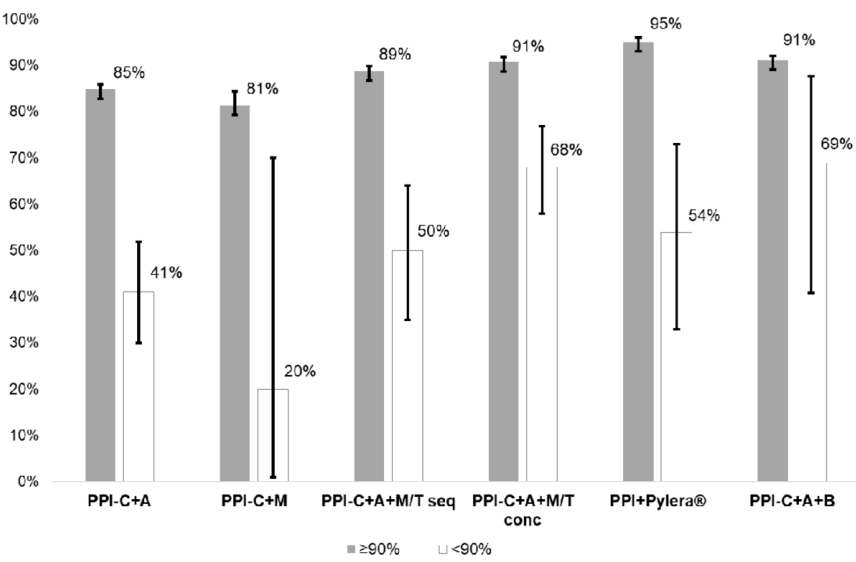

Figure 5 Eradication rate (and $95 \% \mathrm{Cl}$ ) by treatment according to compliance. A, amoxicillin; B, bismuth salts; C, clarithromycin; Conc, concomitant; $\mathrm{M}$, metronidazole; PPI, proton pump inhibitor; Seq, sequential; T, tinidazole. clarithromycin, amoxicillin and bismuth). Additionally, use of the recommended dose of PPI improved cure rates in all treatment categories, although when high instead of standard doses were used, no additional benefit was found as shown in table 4 .

Findings from the present study should be interpreted with caution on account of a number of limitations. First of all, this study is not a randomised controlled clinical trial; therefore, comparisons of treatments must be taken with care, due to unidentified allocation biases that may affect effectiveness. These include different local resistance rates, the age of treatment groups, treatment and care costs for patients and providers, and the use of the mITT analysis versus the traditional ITT (which could overestimate eradication) among others. However, these limitations are inherent to studies focused on clinical practice, which are necessary to elucidate the outcomes in routine practice.

Another drawback is that inclusion rates and numbers varied between centres, regions and countries according to the number of $H$. pylori infections managed in each outpatient clinic. For example, standard clinics in regions with low infection prevalence may obviously attend a lower number of $H$. pylori-infected patients, thus affecting their inclusion rates. Although results may not be fully representative of the general population, it is important to mention that in those highest recruiting countries such as Spain, there was a wide variety of centre types (large hospitals vs small outpatients clinics) and therefore this could balance the distribution and the representativeness of the population. In any case, in our sensitivity analysis, we did not identify any significant bias derived from high versus low inclusion countries. In addition, even if we may think heterogeneity was inevitably present, it is important to highlight that the standard triple therapy did not reach an optimal effectiveness in any of the regions; and, by contrast, in all the regions, all quadruple regimens (concomitant, single capsule bismuth quadruple 


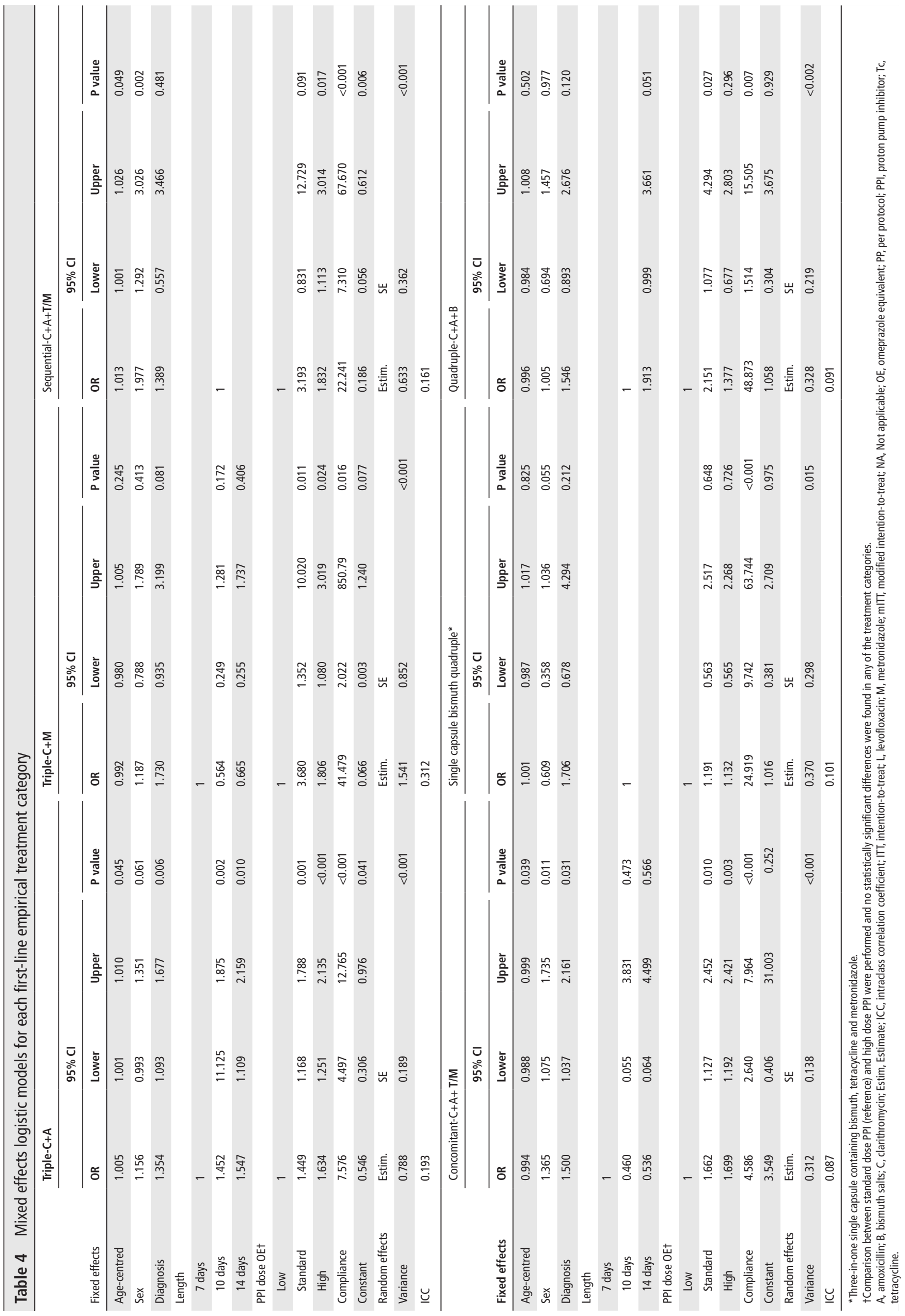


and bismuth-amoxicillin-clarithromycin quadruple therapies) achieved $\approx 90 \%$ eradication rates, which confers consistency to the cluster-by-cluster analysis of the data.

Finally, our intention to study clinical practice forced us to register as many open management options as possible; this increases heterogeneity and sometimes limits the amount of data obtainable from each case. For example, individual antibacterial resistance was available in a relatively low proportion of cases, translating what happens in day-to-day clinical practice in Europe. In the future, PCR testing could ease bacterial antibiotic susceptibility evaluation if such method is shown to be reliable in faecal samples, which would avoid invasive testing such as endoscopy.

In contrast to these limitations, we believe that this type of study has a number of strengths that compensate the weaker areas. The open inclusion criteria ensure that our data represent the real clinical practice of the participant centres, and it allow the evaluation of the widest range of therapeutic options and patient contexts. Although data are heterogeneous, the analyses showed that the measure of the effect is consistent throughout Europe. Moreover, the large number of recruiters and countries has provided, to our knowledge, the largest international prospective series on $H$. pylori treatment under a common research protocol. This has enabled us to perform multivariate analyses to control for confounding variables, data bias and heterogeneity, to develop regional and timetrend approaches. The inclusion of centres with different levels of experience in $\mathrm{H}$. pylori gave us a wide view of real practice. Finally, a high-quality method has been used to register, store, manage and monitor the data by the use of Online Platform for Collaborative Research AEG-REDCap, which provides stability and coherence to the data with programmed and real-time quality controls, queries, reports and statistics.

In light of these results, we may conclude that in order to obtain over $90 \%$ eradication rates consistently, to avoid retreatment and to prevent patient dropout, physicians should be encouraged to use quadruple therapies, because these are the only regimens that consistently achieve eradication rates $\geq 90 \%$. Those treatments were as follows: 14-day non-bismuth quadruple concomitant therapy (PPI, amoxicillin, clarithromycin and metronidazole), 14-day standard triple plus bismuth (PPI, bismuth, amoxicillin and clarithromycin) and 10-day bismuth quadruple therapy (PPI, bismuth, tetracycline and metronidazole). If antibiotic resistance rates are high in the local geographical area, and especially if dual resistance to clarithromycin and metronidazole is greater than $15 \%$, bismuth quadruple therapy may be the most reliable choice.

Prescribing physicians must also take into consideration that regardless of the treatment chosen or the clinical context, compliance with treatment was the most relevant factor for achieving successful eradication, so treatment, procedures and expectations must be carefully explained to the patient.

Although overall $H$. pylori cure rates in the European Registry are relatively disappointing, different regions of Europe are slowly and heterogeneously incorporating recommended practices such as prescribing quadruple therapies for 2 weeks with an increased dose of acid inhibition. The observed ongoing adaptation of real clinical practice to recommendations gives room for hope, especially considering the parallel improvement (up to $10 \%$ in some regions) in overall efficacy in Europe in only 5 years.

\footnotetext{
Author affiliations

${ }_{1}^{1}$ Gastroenterolgy Department, Hospital Universitario de La Princesa, Instituto de Investigación Sanitaria Princesa (IIS-IP), Universidad Autónoma de Madrid (UAM), Centro de Investigacion Biomedica en Red de Enfermedades Hepaticas y Digestivas (CIBEREHD), Madrid, Spain

${ }^{2}$ Department of Pancreatic, Biliary and Upper Digestive Tract Disorders, AS Loginov
} Moscow Clinical Scientific Center, Moscow, Russian Federation
${ }^{3}$ A.I. Yevdokimov Moscow State University of Medicine and Dentistry, Moscow, Russian Federation

${ }^{4}$ Gastroenterology, AM DC Rogaska, Rogaska Slatina, Slovenia

${ }^{5}$ Gastroenterology, Agencia Sanitaria Costa del Sol, Red de Investigación en Servicios de Salud en Enfermedades Crónicas (REDISSEC), Marbella, Spain

${ }^{6}$ Department of of Surgical and Medical Sciences, University of Bologna, Bologna, Italy

${ }^{7}$ Gastroenterology, Hospital Donostia/Instituto Biodonostia, Universidad del País

Vasco (UPV/EHU), CIBEREHD, San Sebastian, Spain

${ }^{8}$ Gastroenterology, Hospital de Valme, CIBEREHD, Sevilla, Spain

${ }^{9}$ Gastroenterololgy, Østfold Hospital Trust, Grålum, Norway

${ }^{10}$ Digestive Diseases Centre GASTRO, Institute of Clinical and Preventive Medicine \&

Faculty of Medicine, University of Latvia, Riga, Latvia

${ }^{11}$ Gastroenterology, Hospital Universitario Central de Asturias, Oviedo, Spain

${ }^{12}$ Gastroenterology, Henry Dunant Hospital, Athens, Greece

${ }^{13}$ Gastroenterology, Lithuanian University of Health Sciences, Kaunas, Lithuania

${ }^{14}$ Gastroenterology, HM Sanchinarro, Madrid, Spain

${ }^{15}$ Internal Medicine, National Medical University named after O.O.Bogomolets, Kyiv, Ukraine

${ }^{16}$ Medicina Interna e Gastroenterologia, Fondazione Policlinico Universitario A.

Gemelli IRCCS, Università Cattolica del Sacro Cuore, Roma, Italy

${ }^{17}$ Internal Medicine/Gastroenterology, Hacettepe University Faculty of Medicine, Ankara, Turkey

${ }^{18}$ Gastroenterology, Leeds General Infirmary, Leeds, UK

${ }^{19}$ Gastroenterology, Ferencváros Health Centre, Budapest, Hungary

${ }^{20}$ i3S - Instituto de Investigação e Inovação em Saúde. Medical Faculty of Porto. Universidade do Porto, Porto, Portugal

${ }^{21}$ Gastroenterology, Rabin Medical Center, Tel Aviv University, Petah Tikva, Israel

${ }^{22}$ Medical Microbiology, Medical University of Sofia, Sofia, Bulgaria

${ }^{23}$ Gastroenterology, Timisoara Hospital, Timisoara, Romania

${ }^{24}$ Gastroenterology, Hepatology \& Nutrition, CHU de Charleroi, Charleroi, Belgium

${ }^{25}$ Gastroenterology, University Hospital of Split, University of Split, Split, Croatia

${ }^{26}$ Gastroenterology, Medical Centre for Postgraduate Education, Warsaw, Poland

${ }^{27}$ Gastroenterology, University Hospital Basel, Basel, Switzerland

${ }^{28}$ Gastroenterology, Hepatology and Infectious Diseases, Otto-von-Guericke

University, Magdeburg, Germany

${ }^{29}$ Clinical Medicine, Zealand University Hospital, Copenhagen University,

Copenhagen, Denmark

${ }^{30}$ Gastroenterology and Hepatology, Meander Medical Center, Amersfoort,

Netherlands

${ }^{31}$ Clinic for Gastroenterology and Hepatology, Clinical Center of Serbia and School of

Medicine, University of Belgrade, Belgrade, Serbia

${ }^{32}$ Internal Medicine, Herttoniemi Hospital, Helsinki, Finland

${ }^{33}$ Gastroenterology, Hospital San Pedro de Alcántara, CIBEREHD, Cáceres, Spain

${ }^{34}$ Gastroenterology, Gastrocentr, Perm, Russian Federation

${ }^{35}$ Gastroenterology, Digestive Ukrainian Academy of Medical Sciences, Kyiv, Ukraine

${ }^{36}$ Gastroenterology, Hospital Clinico Universitario Lozano Blesa, CIBEREHD,

Zaragoza, Spain

${ }^{37}$ Departmento de Psicología Social y Metodología, Facultad de Psicología,

Universidad Autónoma de Madrid, Madrid, Spain

${ }^{38}$ Servicio de Medicina Preventiva, Complejo Hospitalario Universitario de Santiago de Compostela, Santiago de Compostela, Spain

${ }^{39}$ Gastroenterology, Althaia Xarxa Assistencial Universitària de Manresa and

Universitat de Vic-Universitat Central de Catalunya (UViCUCC), Manresa, Spain

${ }^{40}$ Centre Hospitalier Annecy Genevois, Pringy, France

${ }^{41}$ Laboratoire de Bactétiologie, Hôpital Pellegrin, Bordeaux, France

${ }^{42}$ Gastroenterology, Trinity College Dublin, Dublin, Ireland

Correction notice This article has been corrected since it published Online First. The second author's name has been corrected.

Acknowledgements We want to thank Pau Alarcón (Barcelona, Spain) and Jorge Calvo Albinelli, who performed advanced data managing and programming for this project. We want to thank the Spanish Association of Gastroenterology (AEG) for providing the e-CRF service free of charge.

Collaborators Jen Hinojosa (Agencia Sanitaria Costa del Sol Marbella Spain Acquired data, critically reviewed the manuscript draft and approved the submitted manuscript); Inmaculada Santaella (Agencia Sanitaria Costa del Sol Marbella Spain Acquired data, critically reviewed the manuscript draft and approved the submitted manuscript); Nuria Fernandez Moreno (Agencia Sanitaria Costa del Sol Marbella Spain Acquired data, critically reviewed the manuscript draft and approved the submitted manuscript); Ilaria Maria Saracino (University of Bologna Italy Acquired data, critically reviewed the manuscript draft and approved the submitted manuscript); Horacio Alonso Galán (Hospital de Donisti San Sebastian Spain Acquired data, critically reviewed the manuscript draft and approved the submitted manuscript); Almudena Durán (Hospital de la Princesa Madrid Spain Acquired data, critically reviewed the manuscript draft and approved the submitted manuscript); Jennifer Fernandez Pacheco (Hospital de la Princesa Madrid Spain Acquired data, 
critically reviewed the manuscript draft and approved the submitted manuscript); Miroslav Vujasinovic (Slovenj Gradec General Hospital Slovenj Gradec Slovenia Acquired data, critically reviewed the manuscript draft and approved the submitted manuscript); Rinaldo Pellicano Molinette (Hospital Turin Italy Acquired data, critically reviewed the manuscript draft and approved the submitted manuscript); Zdenko Kikec (General Hospital Slovenj Gradec Slovenj Gradec Slovenia Acquired data, critically reviewed the manuscript draft and approved the submitted manuscript); Pedro-Luis Gonzalez Cordero (Hospital San Pedro de Alcantara Caceres Spain Acquired data, critically reviewed the manuscript draft and approved the submitted manuscript): Maia Donday (Hospital de la Princesa, CIBERehd, Instituto de Salud Carlos III Madrid Spain Monitored and interpreted the data, critically reviewed and approved the final manuscript); Angel Lanas (Hospital Clinico Universitario Lozano Blesa Zaragoza Spain Acquired data, critically reviewed the manuscript draft and approved the submitted manuscript); Polona Lampic (Diagnosticni center Bled Bled Slovenia Acquired data, critically reviewed the manuscript draft and approved the submitted manuscript); Vid Leban (Diagnosticni center Bled Bled Slovenia Acquired data, critically reviewed the manuscript draft and approved the submitted manuscript); Aleksander Gruncic Krajnc (Diagnosticni center Bled d.o.o. Bled Slovenia Acquired data, critically reviewed the manuscript draft and approved the submitted manuscript); Natasa Brglez Jurecic (Diagnosticni center Bled d.o.o. Bled Slovenia Acquired data, critically reviewed the manuscript draft and approved the submitted manuscript); Galyna Dmytrivna Fadieienko (Ukrainian Academy of Medical Sciences Kharkiv Ukrania Acquired data, critically reviewed the manuscript draft and approved the submitted manuscript); Lorena Lee (Instituto Fundación Teófilo Hernando Madrid Spain Monitored and interpreted the data, critically reviewed and approved the final manuscript); Irene V Barbado (Instituto Fundación Teófilo Hernando Madrid Spain Monitor and interpret the data, critically reviewed and approved the final manuscript); Alfredo José Lucendo (Hospital General de Tomelloso Tomelloso Spain Acquired data, critically reviewed the manuscript draft and approved the submitted manuscript); Jesus Barrio Rlo Hortega (Hospital Valladolid Spain Acquired data, critically reviewed the manuscript draft and approved the submitted manuscript); Tatiana Alekseevna Ilchishina (SM-clinic Saint-Petersburg Russia Acquired data, critically reviewed the manuscript draft and approved the submitted manuscript); Irina Voynovan (Moscow Scientific Clinical Center Moscow Russia Acquired tdata, critically reviewed the manuscript draft and approved the submitted manuscript); Luis Ignacio Fernández-Salazar (Hospital Clínico Universitario Valladolid Spain Acquired data, critically reviewed the manuscript draft and approved the submitted manuscript); Jose María Huguet (Hospital General Universitario de Valencia Valencia Spain Acquired data, critically reviewed the manuscript draft and approved the submitted manuscript); Pilar Canelles (Hospital General Universitario de Valencia Valencia Spain Acquired data, critically reviewed the manuscript draft and approved the submitted manuscript); Aiman Silkanovna Sarsenbaeva (Gastroenterological center Chelyabinsk Russia Acquired data, critically reviewed the manuscript draft and approved the submitted manuscript); Ines Modolell Consorci (Sanitari Terrassa Terrassa Spain Acquired data, critically reviewed the manuscript draft and approved the submitted manuscript); Pedro Almela (Hospital General Universitario de Castellon Castellon Spain Acquired data, critically reviewed the manuscript draft and approved the submitted manuscript); Marina Roldán Lafuente (Hospital General Universitario de Castellón Castellón de la Plana Spain Acquired data, critically reviewed the manuscript draft and approved the submitted manuscript); Josep Maria Botargues (Hospital Universitari de Bellvitge L'Hospitalet de Llobregat Spain Acquired data, critically reviewed the manuscript draft and approved the submitted manuscript); Miguel Areia (Portuguese Oncology Institute Coimbra Portugal Acquired data, critically reviewed the manuscript draft and approved the submitted manuscript); Luís Elvas (Portuguese Oncology Institute Coimbra Portugal Acquired data, critically reviewed the manuscript draft and approved the submitted manuscript); Susana Isabel Alves (Portuguese Oncology Institute Coimbra Portugal Acquired data, critically reviewed the manuscript draft and approved the submitted manuscript); Daniel Brito (Portuguese Oncology Institute Coimbra Portugal Acquired data, critically reviewed the manuscript draft and approved the submitted manuscript); Ana Teresa Cadime (Portuguese Oncology Institute Coimbra Portugal Acquired data, critically reviewed the manuscript draft and approved the submitted manuscript); Sandra Lúcia Madeira Saraiva (Oncologycal Hospital of Coimbra Coimbra Portugal Acquired data, critically reviewed the manuscript draft and approved the submitted manuscript); Charalampos Tzathas (Tzaneio General Hospital Pireaus Greece Acquired data, critically reviewed the manuscript draft and approved the submitted manuscript); Vassiliki Ntouli (Tzaneio General Hospital Pireaus Greece Acquired data, critically reviewed the manuscript draft and approved the submitted manuscript); Alicia C Marin (Hospital de la Princesa, CIBERehd, Instituto de Salud Carlos III Madrid Spain Monitore and interpretd the data, critically reviewed the manuscript draft and approved the submitted manuscript); Cem Simsek (Hacettepe University Ankara Turkey Acquired data and approved the submitted manuscript); Gerardo Nardone University Federico II Naples Italy Acquired data, critically reviewed the manuscript draft and approved the submitted manuscript); Alba Rocco (University Federico II Naples Italy Acquired data, critically reviewed the manuscript draft and approved the submitted manuscript); Juan Antonio Ortuño (Hospital Universitari i Politècnic La Fe Valencia Spain Acquired data, critically reviewed the manuscript draft and approved the submitted manuscript); Tommaso Di Maira (University Hospital La Fe Valencia
Spain Acquired data, critically reviewed the manuscript draft and approved the submitted manuscript); Sotirios D Georgopoulos (Athens Medical Center, Paleo Faliron General Hospital Athens Greece Acquired data, critically reviewed the manuscript draft and approved the submitted manuscript); Stephan Brackmann (Lovisenberg Diakonale Hospital Oslo Norway Acquired data, critically reviewed the manuscript draft and approved the submitted manuscript); Vendel Kristensen Lovisenberg (Diakonale Hospital Oslo Norway Acquired data, critically reviewed the manuscript draft and approved the submitted manuscript); Blas Jose GomezRodriguez (Hospital Quiron Sagrado Corazon Seville Spain Acquired data, critically reviewed the manuscript draft and approved the submitted manuscript); Perminder Singh Phull (Aberdeen Royal Infirmary Aberdeen UK Acquired data, critically reviewed the manuscript draft and approved the submitted manuscript); Sergey Alekseyevich (Alekseenko Far Eastern State Medical University Khabarovsk Russia Acquired data, critically reviewed the manuscript draft and approved the submitted manuscript); Monica Perona (Hospital Quirón Marbella Spain Acquired data, critically reviewed the manuscript draft and approved the submitted manuscript); Rustam Abdulkhakov (Kazan State Medical University Kazan Russia Acquired data, critically reviewed the manuscript draft and approved the submitted manuscript); Deirdre McNamara (Adelaide \& Meath Hospital Tallaght Dublin Ireland Acquired data, critically reviewed the manuscript draft and approved the submitted manuscript); Sinead M Smith (Trinity College Dublin Dublin Ireland Acquired data, critically reviewed the manuscript draft and approved the submitted manuscript); Denise Elizabeth Brennan (Trinity College Dublin Dublin Ireland Acquired data, critically reviewed the manuscript draft and approved the submitted manuscript); Marina Fedorovna Osipenko (Novosibirsk State Medical University Novosibirsk Russia Acquired data, critically reviewed the manuscript draft and approved the submitted manuscript); Cristobal de la Coba (Hospital de Cabueñes Gijon Spain Acquired data, critically reviewed the manuscripts draft and approved the submitted manuscript); Pilar Varela (Hospital de Cabueñes Gijon Spain Acquired data, critically reviewed the manuscript draft and approved the submitted manuscript); Maria Anatolyevna Livzan (Omsk state medical academy Omsk Russia Acquired data, critically reviewed the manuscript draft and approved the submitted manuscript); Oleg V Zaytsev (First Clinical Medical Center Kovrov Russia Acquired data, critically reviewed the manuscript draft and approved the submitted manuscript); Vladislav Vladimirovich Tsukanov (Federal State Budgetary Institution, Scientific research institute of medical problems of the North Siberian branch under the Russian Academy of Medical Sciences (FSBI 'SRIMPN' SB RAMS) Krasnoyarsk Russia Acquired data, critically reviewed the manuscript draft and approved the submitted manuscript); Alexander Viktorovich Vasyutin (Federal State Budgetary Scientific Institution Scientific research institute of medical problems of the North Krasnoyarsk Russia Acquired data, critically reviewed the manuscripts draft and approved the submitted manuscript); Olga Sergeevna Amelchugova (Federal State Budgetary Scientific Institution Scientific research institute of medical problems of the North Krasnoyarsk Russia Acquired data, critically reviewed the manuscript draft and approved the submitted manuscript); Spiros Michopoulus (Alexandra Hospital Athens Greece Acquired data, critically reviewed the manuscript draft and approved the submitted manuscript); Sergey Gennadievich Burkov (Outpatient clinic Moscow Russia Acquired data, critically reviewed the manuscript draft and approved the submitted manuscript); Dan Dumitrascu (Medical Department 2, University of Medicine and Pharmacy luliu Hatieganu Cluj Napoca Romania Acquired data, critically reviewed the manuscript draft and approved the submitted manuscript); Bogdan lanosi (Medical Department 2, University of Medicine and Pharmacy luliu Hatieganu Cluj Napoca Romania Acquired data, critically reviewed the manuscript draft and approved the submitted manuscript); Ingrid Prytz Berset (Alesund Hospital Alesund Norway Acquired data, critically reviewed the manuscript draft and approved the submitted manuscript); Rafael Ruiz-Zorrilla Lopez (Hospital de Sierrallana Torrelavega Spain Acquired data critically reviewed the manuscript draft and approved the submitted manuscript); Charo Antón (Clinico de Valencia Valencia Spain Acquired data, critically reviewed the manuscript draft and approved the submitted manuscript); Anne Courillon-Mallet (Centre Hospitalier Intercommunal (CIHV) Villeneuve St Georges France Acquired data, critically reviewed the manuscript draft and approved the submitted manuscript); Natasa Brglez Jurecic (Splosna bolnisnica Trbovlje Trbovlje Slovenia Acquired data, critically reviewed the manuscript draft and approved the submitted manuscript); Judith Gomez-Camarero (Hospital Universitario de Burgos Burgos Spain Acquired data, critically reviewed the manuscript draft and approved the submitted manuscript); Manuel Jimenez-Moreno (Hospital Universitario de Burgos Burgos Spain Acquired data, critically reviewed the manuscript draft and approved the submitted manuscript); Ahmet Uygun (GATA Hospital Ankara Turkey Acquired data, critically reviewed the manuscript draft and approved the submitted manuscript); Ian Leonard Phillip Beales (Norfolk and Norwich University Hospital Norwich UK Acquired data, critically reviewed the manuscript draft and approved the submitted manuscript); Alain Huerta-Madrigal (Hospital Universitario Madrid Sanchinarro Madrid Spain Acquired the data, critically reviewed the manuscript draft and approved the submitted manuscript); Javier Alcedo (Hospital de Barbastro Huesca Spain Acquired the data, critically reviewed the manuscript draft and approved the submitted manuscript); Mercè Barenys (Hospital de Viladecans Viladecans Spain Acquired data, critically reviewed the manuscript draft and approved the submitted manuscript); Francesco Franceschi (Catholic University of Rome Rome Italy Acquired data, critically 
reviewed the manuscript draft and approved the submitted manuscript); JeanCharles Delchier (Henri Mondor Hospital Créteil France Acquired data, critically reviewed the manuscript draft and approved the submitted manuscript); Liliana Silvia Pozzati (Mérida Hospital Mérida Spain Acquired data, critically reviewed the manuscript draft and approved the submitted manuscript); Monika Augustyn (Wojskowy Szpital Kliniczny Cracow Poland Acquired data, critically reviewed the manuscript draft and approved the submitted manuscript); Maja Seruga (Hospital Murska Sobota Slovenia Slovenia Acquired data, critically reviewed the manuscript draft and approved the submitted manuscript); Miriam Hiestand (Hospital of Chur Chur Switzerland Acquired data, critically reviewed the manuscript draft and approved the submitted manuscript); Patric Mosler (Hospital of Chur Chur Switzerland Acquired data, critically reviewed the manuscript draft and approved the submitted manuscript); Zaza Beniashvili (Rabin Medical Center, Hospital Ha Sharon Petah Tikva Israel Acquired data, critically reviewed the manuscript draft and approved the submitted manuscript); Doron Boltin (Rabin Medical Center, Beilinson Campus Petah Tikva Israel Acquired data, critically reviewed the manuscript draft and approved the submitted manuscript); Hubert Louis (Hôpital Erasme Brussels Belgium Acquired data, critically reviewed the manuscript draft and approved the submitted manuscript); Ramon Pajares (Hospital Universitario Infanta Sofía San Sebastian de los Reyes Spain Acquired data, critically reviewed the manuscript draft and approved the submitted manuscript); Natalia Valerievna Zakharova (North-western State Medical University named after I.I.Mechnikov St. Petersburg Russia Acquired data, critically reviewed the manuscript draft and approved the submitted manuscript); Natalia Nikolaevna Dekhnich (Institute of Antimicrobial Chemotherapy Smolensk Russia Acquired data, critically reviewed the manuscript draft and approved the submitted manuscript); Victor Asparuhov Kamburov (Lyulin Hospital Sofia Bulgaria Acquired data, critically reviewed the manuscript draft and approved the submitted manuscript); Maria Pina Dore (Clinica Medica, Dipartimento di Scienze Mediche Chirurgiche e Sperimentali, Università di Sassari Sassari Italy Acquired data, critically reviewed the manuscript draft and approved the submitted manuscript); Lorena Sancho (Del val Hospital Rey Juan Carlos Madrid Spain Acquired data, critically reviewed the manuscript draft and approved the submitted manuscript); Noelia Alcaide (Hospital Clínico Universitario Valladolid Spain Acquired the data, critically reviewed the manuscript draft and approved the submitted manuscript); Oscar Núñez (Hospital Universitario Sanitas La Moraleja Madrid Spain Acquired data, critically reviewed the manuscript draft and approved the submitted manuscript); Katrine Dvergsnes Sørlandet (Sykehus HF Kristiansand Norway Acquired data, critically reviewed the manuscript draft and approved the submitted manuscript); Peter Malfertheiner (Otto-von-Guericke University Hospital Magdeburg Germany Acquired data, critically reviewed the manuscript draft and approved the submitted manuscript); Ana Campillo (Hospital Reina Sofia Tudela Spain Acquired data, critically reviewed the manuscript draft and approved the submitted manuscript); Miguel Fernandez-Bermejo (Clinica San Francisco Caceres Spain Acquired data, critically reviewed the manuscript draft and approved the submitted manuscript); Manue Domínguez-Cajal (Hospital San Jorge Huesca Spain Acquired data, critically reviewed the manuscript draft and approved the submitted manuscript); José Luis Domínguez Jiménez (Alto Guadalquivir Hospital Andújar Spain Acquired data, critically reviewed the manuscript draft and approved the submitted manuscript); Alicia Algaba (Hospital Universitario de Fuenlabrada Madrid Spain Acquired data, critically reviewed the manuscript draft and approved the submitted manuscript); Fernando Bermejo (Hospital Universitario de Fuenlabrada Madrid Spain Acquired data, critically reviewed the manuscript draft and approved the submitted manuscript); Borislav Vladimirov (University hospotal Queen Ioanna-Isul Sofia Bulgaria Acquired data, critically reviewed the manuscript draft and approved the submitted manuscript); László Czakó (University of Szeged Szeged Hungary Acquired data, critically reviewed the manuscript draft and approved the submitted manuscript); Teresa Angueira (Hospital General de Tomelloso Ciudad Real Spain Acquired data, critically reviewed the manuscript draft and approved the submitted manuscript); Eduardo lyo (Hospita Comarcal de Inca Mallorca Spain Acquired data, critically reviewed the manuscript draft and approved the submitted manuscript); Ekaterina Yuryevna (Plotnikova Kemerovo State Medical Academy Kuzbass Regional Hepatology Center Kemerovo Russia Acquired data, critically reviewed the manuscript draft and approved the submitted manuscript); Larissa Tarasova (Chuvash State University named after I.N. Ulyanov, the chair of faculty therapy Cheboksary Russia Acquired data, critically reviewed the manuscript draft and approved the submitted manuscript); Ludmila Grigorieva (Chuvash State University named after I.N. Ulyanov, the chair of faculty therapy Cheboksary Russia Acquired data, critically reviewed the manuscript draft and approved the submitted manuscript); Judith Millastre (Hospital de Barbastro Barbastro Spain Acquired data, critically reviewed the manuscript draft and approved the submitted manuscript); Bruno Richard-Molard (Clinique Bordeaux Nord Bordeaux France Acquired data, critically reviewed the manuscript draft and approved the submitted manuscript); Aldis Pukitis Paul Stradins (Clinical University Hospital Riga Latvia Acquired data, critically reviewed the manuscript draft and approved the submitted manuscript); Valeriy Kryvy (Crimean State Medical University named after S.I.Georgievskyi Simferopol Ukrania Acquired data, critically reviewed the manuscript draft and approved the submitted manuscript); Roald Torp (Sykehuset Innlandet, Hamar Hamar Norway Acquired data, critically reviewed the manuscript draft and approved the submitted manuscript); Albert Tomàs (Consorci Sanitari del Garraf Sant
Pere de Ribes Spain Acquired data, critically reviewed the manuscript draft and approved the submitted manuscript); Edurne Amorena (Complejo Hospitalario de Navarra Pamplona Spain Acquired data, critically reviewed the manuscript draft and approved the submitted manuscript); Fermin Estremera (Complejo Hospitalario de Navarra Pamplona Spain Acquired data, critically reviewed the manuscript draft and approved the submitted manuscript); Rossen Nikolov (University Hospital St Ivan Rilski Sofia Bulgaria Acquired data, critically reviewed the manuscript draft and approved the submitted manuscript); Asghar Quasim (Beacon Hospital Dublin Ireland Acquired data, critically reviewed the manuscript draft and approved the submitted manuscript); Yury Aleksandrovich Kucheryavyy (Moscow State University of Medicine and Dentistry named after A.I. Evdokimov Moscow Russia Acquired data, critically reviewed the manuscript draft and approved the submitted manuscript); Natalia Baryshnikova (Fisrt St-Petersburg Pavlov State Medical University St Petersburg Russia Acquired data, critically reviewed the manuscript draft and approved the submitted manuscript); Xavier Calvet (Corporació Sanitària Universitària Parc Taulí. CIBERehd, Instituto de Salud Carlos III Departament de Medicina. Universitat Autònoma de Barcelona Sabadell Spain Acquired data, critically reviewed the manuscript draft and approved the submitted manuscript); Ariadna Figuerola (Corporació Sanitària Universitària Parc Taulí. CIBERehd, Instituto de Salud Carlos III Departament de Medicina. Universitat Autònoma de Barcelona Sabadell Spain Acquired data, critically reviewed the manuscript draft and approved the submitted manuscript); Marco Romano (Università degli Studi della Campania "Luigi Vanvitelli" Napoli Italy Acquired the data, critically reviewed the manuscript draft and approved the submitted manuscript); Antonietta Gerarda Gravina (Università degli Studi della Campania "Luigi Vanvitelli" Napoli Italy Acquired the data, critically reviewed the manuscript draft and approved the submitted manuscript); Oscar Núñez ("Hospital Uni. Sanitas La Moraleja Madrid Spain Acquired data, critically reviewed the manuscript draft and approved the submitted manuscript); Fazia Mana (UZ Brussel Jette Belgium Acquired the data, critically reviewed the manuscript draft and approved the submitted manuscript); Pilar Sánchez-Pobre (Hospital Clinico San Carlos Madrid Spain Acquired data, critically reviewed the manuscript draft and approved the submitted manuscript); Zoya Spassova (University Hospital St. Ivan Rilski Sofia Bulgaria Acquired data, critically reviewed the manuscript draft and approved the submitted manuscript); Jesús M González-Santiago (Hospital Clínico Universitario. Instituto de Investigación Biomédica de Salamanca (IBSAL). Salamanca Spain Acquired data, critically reviewed the manuscript draft and approved the submitted manuscript); Ricardo Marcos-Pinto (1. Centro Hospitalar do Porto 2. Institute of Biomedical Sciences Abel Salazar, University of Porto 3. CINTESIS, University of Porto Porto Portugal Acquired data, critically reviewed the manuscript draft and approved the submitted manuscript); F Wolfhagen (Albert Schweitze Ziekenhuis Dordrecht The Netherlands Acquired data, critically reviewed the manuscript draft and approved the submitted manuscript); Svetlana Cui (Pauls Stradins Clinical University Hospital Riga Latvia Acquired data, critically reviewed the manuscript draft and approved the submitted manuscript); Ivonne Leeuwenburgh (Sint Franciscus Gasthuis Rotterdam The Netherlands Acquired ata, critically reviewed the manuscript draft and approved the submitted manuscript); Driffa Moussata (Lyon Sud Hospital Pierre Benite France Acquired data, critically reviewed the manuscript draft and approved the submitted manuscript); Adi Lahat-zok (Chaim Sheba medical center and Sackler School of Medicine, Tel Aviv University Israel Acquired data, critically reviewed the manuscript draft and approved the submitted manuscript); Sergii Hryhorovych (Melashchenko Vinnitsa National Medical University Vinnytsia Ukrania Acquired data, critically reviewed the manuscript draft and approved the submitted manuscript); Rasmus Goll (University Hospital of North Norway Tromso Norway Acquired data, critically reviewed the manuscript draft and approved the submitted manuscript); Tatyana Vasilyevna (Zhestkova Ryazan State Medical University Ryazan Russia Acquired data, critically reviewed the manuscript draft and approved the submitted manuscript); Juris Pokrotnieks (Pauls Stradins Clinical University Hospital Riga Latvia Acquired data, critically reviewed the manuscript draft and approved the submitted manuscript); Philippe Émile (Houcke Hôpital Claude Huriez (CHRU) Lille France Acquired data, critically reviewed the manuscript draft and approved the submitted manuscript); Nadiya Byelyayeva (Donetsk National Medical University Donetsk Ukrania Acquired data, critically reviewed the manuscript draft and approved the submitted manuscript); Marta Lozano (Lanagran Hospital Quuiron Malaga Spain Acquired data, critically reviewed the manuscript draft and approved the submitted manuscript); Mette Wildner-Christensen (Odense University Hospital (OUH) Svendborg Denmark Acquired data, critically reviewed the manuscript draft and approved the submitted manuscript); Bengt Odman (Soder Hospital Stockholm Sweden Acquired data, critically reviewed the manuscript draft and approved the submitted manuscript); Yana Valerieva (University Hospital Tsaritsa Yoanna-ISUL Sofia Bulgaria Acquired data, critically reviewed the manuscript draft and approved the submitted manuscript); Alenka Forte (MC Heliks, d.o.o. Trbovlje Slovenia Acquired data, critically reviewed the manuscript draft and approved the submitted manuscript); Antonio Cuadrado (Lavin Hospital Universitario Marqués de Valdecilla Santander Spain Acquired data, critically reviewed the manuscript draft and approved the submitted manuscript); Patrice Pienkowski (Hospital Center de Montauban Montauban France Acquired data, critically reviewed the manuscript draft and approved the submitted manuscript); Ilze Kikuste (University of Latvia, Digestive diseases centre GASTRO Riga Latvia Acquired data, critically reviewed the 
manuscript draft and approved the submitted manuscript); Dag Arne Lihaug Hoff (Aalesund Hospital Aalesund Norway Acquired data, critically reviewed the manuscript draft and approved the submitted manuscript); Jane Moeller Hansen (Odense University Hospital Odense Denmark Acquired data, critically reviewed the manuscript draft and approved the submitted manuscript); Konrads Funka (Digestive Diseases Centre GASTRO Riga Latvia Acquired data, critically reviewed the manuscript draft and approved the submitted manuscript); Alla Kononova (Department of General Practice Tver Russia Acquired data, critically reviewed the manuscript draft and approved the submitted manuscript); Sergey Kolbasnikov (The Head of Department of General Practice Tver Russia Acquired data, critically reviewed the manuscript draft and approved the submitted manuscript); Michael Selgrad (Otto-von Guericke University Magdeburg Germany Acquired data, critically reviewed the manuscript draft and approved the submitted manuscript); Jolanta Sumskiene (Lithuanian University of Health Sciences Hospital Lithuania Lithuania Acquired data, critically reviewed the manuscript draft and approved the submitted manuscript); Jonathan Hirsch (Meir Medical Center Kfar Saba Israel Acquired data, critically reviewed the manuscript draft and approved the submitted manuscript); Francisco Javier Zozaya Larequi (Hospital Universitario Donostia San Sebastián Spain Acquired data, critically reviewed the manuscript draft and approved the submitted manuscript); Alain C Burette (Chirec clinique de la Basilique and clinique Edith Cavell Brussels Belgium Acquired data, critically reviewed the manuscript draft and approved the submitted manuscript); Nora Dancs Petz-Aladar (Teaching Hospital Gyor Hungary Acquired data, critically reviewed the manuscript draft and approved the submitted manuscript); Janne Rajala Herttoniemi (Hospital Helsinky Finland Acquired data, critically reviewed the manuscript draft and approved the submitted manuscript); Christina Reimer (Hvidovre Hospital Hvidovre Denmark Acquired data, critically reviewed the manuscript draft and approved the submitted manuscript); Diogo Libanio (Gastroenterology Department Instituto Portugues de Oncologia do Porto (IPO-Porto) Porto Portugal Acquired data, critically reviewed the manuscript draft and approved the submitted manuscript); Pedro Pimentel-Nunes (Gastroenterology Department Instituto Portugues de Oncologia do Porto (IPO-Porto) Porto Portugal Acquired the data, critically reviewed the manuscript draft and approved the submitted manuscript); Ivailo Evstatiev (University Hospital St Ekaterina Sofia Bulgaria Acquired data, critically reviewed the manuscript draft and approved the submitted manuscript); Juozas Kupcinskas (Lithuanian University of Health Sciences Kaunas Lithuania Acquired data, critically reviewed the manuscript draft and approved the submitted manuscript); Mikhail Butov (State Budgetary Educational Institution of Higher Professional Education Ryazan State I.P. Pavlov Medical University of the Ministry of Public Health of Russian Federation (SB?IHPE RSMU of Ministry of Public Health of Russian Federation) Department of Propaedeutics of Internal Diseases Ryazan Russia Acquired data, critically reviewed the manuscript draft and approved the submitted manuscript): Peter Mensink (Medisch Spectrum Twente Enschede The Netherlands Acquired data, critically reviewed the manuscript draft and approved the submitted manuscript); T Tang (IJsselland Ziekenhuis Capelle aan de ljssel The Netherlands Acquired data, critically reviewed the manuscript draft and approved the submitted manuscript); Andrey Yurevich Baranovsky (North-Western State Medical University named after I.I.Mechnicov Saint-Petersburg Russia Acquired data, critically reviewed the manuscript draft and approved the submitted manuscript); Natalya Marchenko (North-Western State Medical University named after I.I. Mechnikov Saint-Patarsburg Russia Acquired data, critically reviewed the manuscript draft and approved the submitted manuscript); Boris Bastens (CHC Liege Liege Belgium Acquired data, critically reviewed the manuscript draft and approved the submitted manuscript); Lyudmila Mateva ("University Hospital St. Ivan Rilski Sofia Bulgaria Acquired data, critically reviewed the manuscript draft and approved the submitted manuscript); Dominique Lamarque (Hôpital Ambroise-Paré Boulogne-Billancourt (Paris) France Acquired data, critically reviewed the manuscript draft and approved the submitted manuscript); Leonardo Henry (Eusebi Department of Medical and Surgical Sciences (DIMEC), University of Bologna, Gastroenterology and Endoscopy Unit, Policlinico S. Orsola-Malpighi Bologna Italy Acquired the data, critically reviewed the manuscript draft and approved the submitted manuscript); Mario Ribeiro (FMUP/IPATIMUP Porto Portugal Acquired data, critically reviewed the manuscript draft and approved the submitted manuscript): $M$ ter Borg (Maxima Medisch Centrum Eindhoven The Netherlands Acquired data, critically reviewed the manuscript draft and approved the submitted manuscript); Alexander C Ford (Leeds Teaching Hospitals Trust Leeds UK Acquired data, critically reviewed the manuscript draft and approved the submitted manuscript); Enrique Medina (Consorcio Hospital General de Valencia Valencia Spain Acquired data, critically reviewed the manuscript draft and approved the submitted manuscript); Manuel Rodriguez-Tellez (Virgen Macarena University Hospital Sevilla Spain Acquired the data, critically reviewed the manuscript draft and approved the submitted manuscript); Francisco José Rancel (Medina Hospital Virgen del Puerto Plasencia Spain Acquired the data, critically reviewed the manuscript draft and approved the submitted manuscript); Elisa Martin (Hospital Virgen del Puerto Plasencia Spain Acquired data, critically reviewed the manuscript draft and approved the submitted manuscript); Carolina Torres Gonzalez (Hospital de la Ribera Alcira (Valencia) Spain Acquired data, critically reviewed the manuscript draft and approved the submitted manuscript); Lissa Maria Franco (Hospital de Cabueñes Oviedo Spain
Acquired data, critically reviewed the manuscript draft and approved the submitted manuscript)

Contributors OPN: Scientific Director and member of the project's Scientific Committee, planned and coordinated the study, designed and programmed the electronic case report form, analysed the data, wrote the manuscript drafts and approved the submitted manuscript. DSB, BT, FL, ML, TR, LK, OS, AG, HS, ATRA, GMB, YN, VL, AT, MV, PB, LGC, TM and VM: acted as national coordinators for their country and as recruiters. Selected national recruiters, collected and helped interpreting data, critically reviewed the manuscript drafts, and approved the submitted manuscript. JCM, AG, KP and CB: acted as non-recruiting national coordinators. Selected nationa recruiters, helped interpreting data, critically reviewed the manuscript drafts and approved the submitted manuscript. AP-A, DV, LB, MC, LR, JP-L, LL, GF, JM-I, LV, GF and IA: collected and helped interpreting data, critically reviewed the manuscripts' drafts and approved the submitted manuscript. MC and AG: supervised, coordinated and monitored data collection, interpreted data, critically reviewed the manuscript drafts and approved the submitted manuscript. JG and CF: supervised, analysed and interpreted data, critically reviewed the manuscript drafts and approved the submitted manuscript. IP: Scientific Director and member of the project's Scientific Committee, critically reviewed the manuscript drafts and approved the submitted manuscript. FM: French National Coordinator and Member of the project's Scientific Committee, designed the protocol, planned the study, analysed the data, critically reviewed the manuscript drafts and approved the submitted manuscript. CO'M: Irish National Coordinator and Member of the project's Scientific Committee, designed the protocol, planned the study, collected and analysed the data, critically reviewed the manuscript drafts, and approved the submitted manuscript. JPG: directed the project and the project's Scientific Committee, obtained funding, designed the protocol and planned the study, acted as Spanish National Coordinator, analysed and interpreted the data, collected patients, critically reviewed the manuscript drafts and approved the final submitted manuscript.

Funding This project has been funded by the European Helicobacter and Microbiota Study Group (EHMSG), the Asociación Española de Gastroenterología (AEG) and the Centro de Investigación Biomédica en Red de Enfermedades Hepáticas y Digestivas (CIBERehd).

Competing interests JPG has served as speaker, consultant and advisory member for or has received research funding from Mayoly, Allergan and Diasorin. OPN has received a research grant from Allergan. JM-I has served as a consultant for Casen Recordati and has received retribution from Allergan for formative actions. MC-F has received retribution from Allergan for formative actions. PB has served as speaker, consultant and advisory member for or has received research funding from Almirall, Allergan and Reckitt Benckiser. APA has received retribution from Allergan and Mylan for formative actions. DSB has served as lecturer for Astellas, AstraZeneca, KRKA and Abbott.

Patient and public involvement Patients and/or the public were not involved in the design, or conduct, or reporting or dissemination plans of this research.

Patient consent for publication Not required.

Ethics approval CEIM Hospital Universitario de La Princesa. There are two approvals: Hp-EuReg 20-Dec-2012 and Hp-EuReg 15-Jan-2015.

Provenance and peer review Not commissioned; externally peer reviewed.

Data availability statement Data are available on reasonable request. All data relevant to the study are included in the article or uploaded as supplementary information.

\section{ORCID iDs}

Dmitry Bordin http://orcid.org/0000-0003-2815-3992

Jose Carlos Machado http://orcid.org/0000-0003-4741-8415

Javier P Gisbert http://orcid.org/0000-0003-2090-3445

\section{REFERENCES}

1 Current European concepts in the management of Helicobacter pylori infection. The Maastricht consensus report. European Helicobacter pylori Study Group. Gut 1997:41:8-13

2 Malfertheiner $\mathrm{P}$, Mégraud F, O'Morain C, et al. Current concepts in the management of Helicobacter pylori infection--the Maastricht 2-2000 Consensus Report. Aliment Pharmacol Ther 2002;16:167-80

3 Malfertheiner P, Megraud F, O'Morain C, et al. Current concepts in the management of Helicobacter pylori infection: the Maastricht III consensus report. Gut 2007:56:772-81.

4 Malfertheiner P, Megraud F, O'Morain CA, et al. Management of Helicobacter pylori infection--the Maastricht IV/ Florence Consensus Report. Gut 2012:61:646-64.

5 Malfertheiner P, Megraud F, O'Morain CA, et al. Management of Helicobacter pylori infection-the Maastricht V/Florence consensus report. Gut 2017;66:6-30.

6 Fallone CA, Chiba N, van Zanten SV, et al. The Toronto consensus for the treatment of Helicobacter pylori infection in adults. Gastroenterology 2016;151:51-69. e14. 
7 Graham DY. Helicobacter pylori eradication therapy research: ethical issues and description of results. Clin Gastroenterol Hepatol 2010;8:1032-6.

8 Graham DY, Lu H, Yamaoka Y. A report card to grade Helicobacter pylori therapy. Helicobacter 2007:12:275-8.

9 Graham DY, Lee Y-C, Wu M-S. Rational Helicobacter pylori therapy: evidencebased medicine rather than medicine-based evidence. Clin Gastroenterol Hepatol 2014;12:177-86. e173; Discussion e112-173.

10 Megraud F, Coenen S, Versporten A, et al. Helicobacter pylori resistance to antibiotics in Europe and its relationship to antibiotic consumption. Gut 2013;62:34-42.

11 Gisbert JP, Calvet X, O'Connor A, et al. Sequential therapy for Helicobacter pylori eradication: a critical review. J Clin Gastroenterol 2010:44:313-25.

12 Gisbert JP, Calvet X. Review article: non-bismuth quadruple (concomitant) therapy for eradication of Helicobater pylori. Aliment Pharmacol Ther 2011;34:604-17.

13 Nyssen OP, McNicholl AG, Megraud F, et al. Sequential versus standard triple first-line therapy for Helicobacter pylori eradication. Cochrane Database Syst Rev 2016;6:CD009034

14 Malfertheiner P. Infection: bismuth improves PPI-based triple therapy for H. pylori eradication. Nat Rev Gastroenterol Hepatol 2010;7:538-9.

15 Liao J, Zheng Q, Liang X, et al. Effect of fluoroquinolone resistance on 14-day levofloxacin triple and triple plus bismuth quadruple therapy. Helicobacter 2013;18:373-7.

16 Ford AC, Malfertheiner P, Giguere M, et al. Adverse events with bismuth salts for Helicobacter pylori eradication: systematic review and meta-analysis. World $J$ Gastroenterol 2008;14:7361-70.

17 Lu H, Zhang W, Graham DY. Bismuth-containing quadruple therapy for Helicobacter pylori: lessons from China. Eur J Gastroenterol Hepatol 2013;25:1-1140.

18 Sun Q, Liang X, Zheng Q, et al. High efficacy of 14-day triple therapy-based, bismuthcontaining quadruple therapy for initial Helicobacter pylori eradication. Helicobacter 2010;15:233-8.

19 Calvet X, García N, López T, et al. A meta-analysis of short versus long therapy with a proton pump inhibitor, clarithromycin and either metronidazole or amoxycillin for treating Helicobacter pylori infection. Aliment Pharmacol Ther 2000;14:603-9.

20 McNicholl AG, Linares PM, Nyssen OP, et al. Meta-Analysis: esomeprazole or rabeprazole vs. first-generation pump inhibitors in the treatment of Helicobacter pylor infection. Aliment Pharmacol Ther 2012;36:414-25.

21 Li B-Z, Threapleton DE, Wang J-Y, et al. Comparative effectiveness and tolerance of treatments for Helicobacter pylori: systematic review and network meta-analysis. BMJ 2015:351:h4052.

22 Grimshaw JM, Russell IT. Effect of clinical guidelines on medical practice: a systematic review of rigorous evaluations. Lancet 1993:342:1317-22.

23 Damschroder LJ, Aron DC, Keith RE, et al. Fostering implementation of health services research findings into practice: a consolidated framework for advancing implementation science. Implement Sci 2009;4:50.

24 Thamer M, Ray NF, Henderson SC, et al. Influence of the NIH consensus conference on Helicobacter pylori on physician prescribing among a Medicaid population. Med Care 1998;36:646-60

25 Grimshaw JM, Russell IT. Achieving health gain through clinical guidelines II: ensuring guidelines change medical practice. Qual Health Care 1994;3:45-52.
26 McNicholl AG, O'Morain CA, Megraud F, et al. As scientific Committee of the Hp-Eureg on behalf of the National $\mathrm{C}$. protocol of the European registry on the management of Helicobacter pylori infection (Hp-EuReg). Helicobacter 2019;24:e12630.

27 Kirchheiner J, Glatt S, Fuhr U, et al. Relative potency of proton-pump inhibitorscomparison of effects on intragastric pH. Eur J Clin Pharmacol 2009;65:19-31.

28 Graham DY, Lu H, Dore MP. Relative potency of proton-pump inhibitors, Helicobacter pylori therapy cure rates, and meaning of double-dose PPi. Helicobacter 2019;24:e12554.

29 Graham DY, Tansel A. Interchangeable use of proton pump inhibitors based on relative potency. Clin Gastroenterol Hepatol 2018;16:800-8. e807.

30 Venerito M, Krieger T, Ecker T, et al. Meta-Analysis of bismuth quadruple therapy versus clarithromycin triple therapy for empiric primary treatment of Helicobacter pylori infection. Digestion 2013;88:33-45.

31 Gisbert JP, Calvet X. Review article: the effectiveness of standard triple therapy for Helicobacter pylori has not changed over the last decade, but it is not good enough. Aliment Pharmacol Ther 2011;34:1255-68.

32 Zagari RM, Romano M, Ojetti V, et al. Guidelines for the management of Helicobacter pylori infection in Italy: the III Working group consensus report 2015. Dig Liver Dis 2015;47:903-12.

33 Song Z-Q, Zhou L-Y. Hybrid, sequential and concomitant therapies for Helicobacter pylori eradication: a systematic review and meta-analysis. World J Gastroenterol 2016:22:4766-75.

34 de Boer WA, Driessen WM, Potters VP, et al. Randomized study comparing 1 with 2 weeks of quadruple therapy for eradicating Helicobacter pylori. Am J Gastroenterol 1994;89:1993-7

35 Gisbert JP MA. Eradication of Helicobacter pylori infection with non-bismuth quadruple concomitant therapy. In: Frontiers in Anti-infective Drug Discovery, 2020: 1-34.

36 Mégraud F. The challenge of Helicobacter pylori resistance to antibiotics: the comeback of bismuth-based quadruple therapy. Therap Adv Gastroenterol 2012;5:103-9.

37 Malfertheiner P, Bazzoli F, Delchier J-C, et al. Helicobacter pylori eradication with a capsule containing bismuth subcitrate potassium, metronidazole, and tetracycline given with omeprazole versus clarithromycin-based triple therapy: a randomised, open-label, non-inferiority, phase 3 trial. Lancet 2011:377:905-13.

38 Saleem A, Qasim A, O'Connor HJ, et al. Pylera for the eradication of Helicobacter pylori infection. Expert Rev Anti Infect Ther 2009:7:793-9.

39 Nyssen OP, McNicholl AG, Gisbert JP. Meta-Analysis of three-in-one single capsule bismuth-containing quadruple therapy for the eradication of Helicobacter pylori. Helicobacter 2019;24:e12570.

40 Gisbert JP, Barrio J, Modolell I, et al. Helicobacter pylori first-line and rescue treatments in the presence of penicillin allergy. Dig Dis Sci 2015;60:458-64.

41 McNicholl AG, Bordin DS, Lucendo A, et al. Combination of Bismuth and Standard Triple Therapy Eradicates Helicobacter pylori Infection in More than $90 \%$ of Patients. Clin Gastroenterol Hepatol 2020;18:89-98.

42 Gisbert JP, McNicholl AG. Optimization strategies aimed to increase the efficacy of $\mathrm{H}$. pylori eradication therapies. Helicobacter 2017;22:hel.12392. 